\title{
Searching for Cool Dust. II. Infrared Imaging of The OH/IR Supergiants, NML Cyg, VX Sgr, S Per, and the Normal Red Supergiants RS Per and T Per*
}

\author{
Michael S. Gordon $^{1}$ (D), Roberta M. Humphreys ${ }^{1}$ (D), Terry J. Jones ${ }^{1}$ (D), Dinesh Shenoy ${ }^{1}$ (D), Robert D. Gehrz ${ }^{1}$ (D) , L. Andrew Helton ${ }^{2}$, \\ Massimo Marengo ${ }^{3}$ (D), Philip M. Hinz ${ }^{4}$, and William F. Hoffman ${ }^{4}$ \\ ${ }^{1}$ Minnesota Institute for Astrophysics, School of Physics and Astronomy 116 Church Street SE, University of Minnesota, Minneapolis, MN 55455, USA \\ gordon@astro.umn.edu \\ ${ }^{2}$ USRA-SOFIA Science Center, NASA Ames Research Center, Moffett Field, CA 94035, USA \\ ${ }^{3}$ Department of Physics and Astronomy, Iowa State University, Ames, IA 50011, USA \\ ${ }^{4}$ Department of Astronomy/Steward Observatory, University of Arizona, 933 North Cherry Avenue, Tucson, AZ 85721, USA \\ Received 2017 July 31; revised 2018 March 21; accepted 2018 March 21; published 2018 April 25
}

\begin{abstract}
New MMT/MIRAC (9-11 $\mu \mathrm{m})$, SOFIA/FORCAST (11-37 $\mu \mathrm{m})$, and Herschel/PACS (70 and $160 \mu \mathrm{m})$ infrared (IR) imaging and photometry is presented for three famous OH/IR red supergiants (NML Cyg, VX Sgr, and S Per) and two normal red supergiants (RS Per and T Per). We model the observed spectral energy distributions (SEDs) using radiative-transfer code DUSTY. Azimuthal average profiles from the SOFIA/FORCAST imaging, in addition to dust mass distribution profiles from DUSTY, constrain the mass-loss histories of these supergiants. For all of our observed supergiants, the DUSTY models suggest that constant mass-loss rates do not produce enough dust to explain the observed infrared emission in the stars' SEDs. Combining our results with Shenoy et al. (Paper I), we find mixed results with some red supergiants showing evidence for variable and high mass-loss events while others have constant mass loss over the past few thousand years.
\end{abstract}

Key words: stars: individual (NML Cyg, RS Per, S Per, T Per, VX Sgr) - stars: mass-loss

\section{Introduction}

The evolution and fate of massive stars depends on mass loss and their mass loss histories. The majority of massive stars $\left(\geqslant 9 M_{\odot}\right)$ will pass through the red supergiant (RSG) stage, long recognized as an important end product of stellar evolution. Recently, Smartt et al. (2009) and Smartt (2015) have suggested that RSGs with initial masses greater than $18 M_{\odot}$ do not explode as supernovae, but may evolve back to warmer temperatures before the terminal explosion or collapse directly to black holes. The RSG stage is also a high mass-losing stage, and to what extent mass loss can affect the terminal state of the RSGs is now an open question. Even though the mass-loss mechanism for RSGs is still debated, we can measure the mass lost from the thermal infrared (IR) emission from dust in the circumstellar ejecta surrounding the RSGs.

In our first paper (Shenoy et al. 2016; hereafter, Paper I), we examined the cold dust in the mid- to far-IR and the mass-loss histories of the famous hypergiants $\mu$ Cep, VY CMa, IRC +10420 , and $\rho$ Cas, whose mass-loss rates are among the highest observed. In this paper, we present similar observations of three strong IR and maser sources, the OH/IR RSGs NML Cyg, VX Sgr, and S Per, plus the normal RSGs RS Per and T Per. OH/IR stars, characterized by strong winds and $\mathrm{OH}$ maser emission, are bright IR sources due to thermal dust emission by their own circumstellar ejecta. The more typical RSGs, without $\mathrm{OH}$ or $\mathrm{H}_{2} \mathrm{O}$ maser emission, also show high mass-loss rates that increase as a function of

\footnotetext{
* Based on observations obtained with: (1) the NASA/DLR Stratospheric Observatory for Infrared Astronomy (SOFIA). SOFIA is jointly operated by the Universities Space Research Association, Inc. (USRA), under NASA contract NAS2-97001, and the Deutsches SOFIA Institut (DSI) under DLR contract 50 OK 0901 to the University of Stuttgart; and (2) the MMT Observatory on Mt. Hopkins, AZ, a joint facility of the Smithsonian Institution and the University of Arizona.
}

luminosity (Reimers 1975; de Jager et al. 1988; Mauron \& Josselin 2011). In this study, we analyze the mass loss in these five RSGs through observations in the mid-IR with SOFIA/ FORCAST (Herter et al. 2012) 11-37 $\mu \mathrm{m}$ imaging, combined with publicly available Herschel $^{5}$ (Pilbratt et al. 2010) PACS (Poglitsch et al. 2010) images. We also include sub-arcsecond resolution $8-12 \mu \mathrm{m}$ observations of NML Cyg (Schuster et al. 2009), S Per, and T Per made with MMT/MIRAC (Hoffmann et al. 1998; Hinz et al. 2000).

Finally, we present spectral energy distribution (SED) models from the radiative-transfer code DUSTY (Ivezic et al. 1997). These SED models, in combination with azimuthal profiles from FORCAST and MIRAC, provide estimates on mass-loss rates, ejecta dust temperatures, and mass-loss histories.

\section{Observations and Data Reduction}

\subsection{SOFIA/FORCAST: Far-IR Imaging $(11-37 \mu \mathrm{m})$}

The targets were observed with SOFIA/FORCAST during Cycles 3 \& 4 (OBS IDs: 03_0082, 04_0013; PI: R. M. Humphreys). FORCAST is a dual-channel mid-IR imager covering the $5-40 \mu \mathrm{m}$ range. Each channel uses a $256 \times 256$ pixel blocked-impurity-band $(\mathrm{BiB})$ array and provides a distortion-corrected $3 ! 2 \times 3 ! 2$ field of view with a scale of $0 . / 768 \mathrm{pix}^{-1}$. FORCAST achieves near-diffractionlimited imaging, with a PSF FWHM of $\sim 3$ !"7 in the longest filters. We elected to image in single-beam mode to maximize

\footnotetext{
5 Herschel is an ESA space observatory with science instruments provided by the European-led Principal Investigator consortia and with important participation from NASA. The Herschel data used in this paper are from the Level 2 (flux-calibrated) images provided by the Herschel Science Center via the NASA/IPAC Infrared Science Archive (IRSA), which is operated by the Jet Propulsion Laboratory, California Institute of Technology, under contract with NASA.
} 
Table 1

Summary of Observations

\begin{tabular}{|c|c|c|c|c|c|}
\hline Target & Instrument & $\begin{array}{l}\text { Date } \\
\text { (UT) }\end{array}$ & $\begin{array}{l}\text { Filter }^{\mathrm{a}} \\
(\mu \mathrm{m})\end{array}$ & $\begin{array}{l}\text { Int Time } \\
\text { (s) }\end{array}$ & $\begin{array}{l}\text { PSF FWHM } \\
\left({ }^{\prime}\right. \\
\left(^{\prime \prime}\right)\end{array}$ \\
\hline \multirow[t]{2}{*}{ VX Sgr } & FORCAST & 2015 Jun 13 & F111, F197, F253 & $77,81,232$ & $2.8,2.9,2.8$ \\
\hline & & & F315, F348, F371 & $224,354,496$ & $3.1,3.4,3.6$ \\
\hline \multirow[t]{3}{*}{ S Per } & MIRAC4 & 2006 Nov 05 & $8.9,9.8$ & 420,60 & $0.3,0.4$ \\
\hline & FORCAST & 2015 Feb 04 & F197, F253 & 164,374 & $2.7,3.2$ \\
\hline & & & F315, F348, F371 & $374,705,1430$ & $3.4,3.6,3.8$ \\
\hline RS Per & FORCAST & 2016 Feb 18 & F197, F315, F371 & $29,270,1062$ & $2.6,3.1,3.6$ \\
\hline \multirow[t]{2}{*}{ T Per } & MIRAC4 & 2009 Oct 02 & $8.9,9.8$ & 50,70 & $0.32,0.35$ \\
\hline & FORCAST & 2016 Sep 17 & F197, F315 & 160,484 & $2.6,3.1$ \\
\hline \multirow[t]{3}{*}{ NML Cyg ${ }^{\mathrm{c}}$} & MIRAC3 & 2006 Jul 23 & $8.9,9.8,11.9$ & $260,260,220$ & $0.3,0.4,0.6$ \\
\hline & FORCAST & 2015 Sep 11 & F197, F253 & 65,233 & $2.6,2.8$ \\
\hline & & & F315, F348, F371 & $320,582,342$ & $3.2,3.4,3.6$ \\
\hline
\end{tabular}

Notes.

${ }^{\mathrm{a}}$ The effective wavelengths of the SOFIA/FORCAST filters are: F197 $=19.7 \mu \mathrm{m}, \mathrm{F} 253=25.3 \mu \mathrm{m}, \mathrm{F} 315=31.5 \mu \mathrm{m}, \mathrm{F} 348=34.8 \mu \mathrm{m}, \mathrm{F} 371=37.1 \mu \mathrm{m}$.

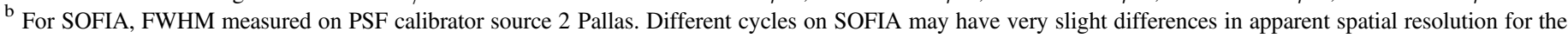
same filter sets. For MIRAC, FWHM measured on $\beta$ And.

${ }^{c}$ NML Cyg MIRAC3/BLINC observations originally published in Schuster et al. (2009).

throughput. The observations were obtained in the standard two-position chop-and-nod mode with the direction of the nod matching the direction of the chop (NMC). The data were reduced by the SOFIA Science Center using the FORCAST Redux pipelines version 1.0.3 (S Per), 1.0.5 (VX Sgr), 1.0.7 (NML Cyg), and 1.1.0 (RS Per, T Per). After correcting for bad pixels and droop effects, the pipeline removed sky and telescope background emission by first subtracting chopped image pairs and then subtracting nodded image pairs. The resulting positive images were aligned and merged. The details of the FORCAST pipeline are discussed in the Guest Investigator Handbook for FORCAST Data Products, Rev. A3. ${ }^{6}$ The FORCAST and MIRAC observations are summarized in Table 1.

Bright point sources cause cross-talk in the horizontal direction on the FORCAST array. To mitigate this effect, chop angles were selected so that the cross-talk pattern from one chop position did not overlap with the other chop position. Additionally, the FORCAST pipeline applies a correction that reduces the effect, although some of the pattern remains for some targets. The effect is strongest for the brightest IR targets, NML Cyg and VX Sgr; it is less so for S Per and is not present in the images of RS Per or $\mathrm{T}$ Per. However, one effect that may appear in some of the fainter targets (especially T Per) is possible coma introduced from the NMC chopping pattern. ${ }^{7}$ This effect may explain the asymmetries in the surface brightness profile of T Per, shown in Figure 10 and discussed in Section 3.4.

For each of the stars, observations of the asteroid 2 Pallas were used for PSF calibration in the same filters with the fourposition slide in either the mirror position (for the short wavelength channel) or the open position (for the longwavelength channel). The color temperature of the asteroid Pallas $(\sim 160 \mathrm{~K})$ is far less than the effective temperatures of the target stars ( $\gtrsim 3200 \mathrm{~K})$. This color difference is not ideal for a PSF calibrator, as the cooler source will peak at longer wavelengths, possibly resulting in a broader profile. However, Pallas was the only source observed in each SOFIA cycle under

\footnotetext{
6 Available at https://www.sofia.usra.edu/researchers/data-products.

7 See description of optical aberrations in Section 1.3.1 of the SOFIA Observer's Handbook, available at https://www.sofia.usra.edu/science/ proposing-and-observing/sofia-observers-handbook-cycle-6.
}

the same conditions and at each wavelength studied in this work. We analyzed another calibrator ( $\alpha$ Aur) at 11.1 and $31.5 \mu \mathrm{m}$ and measured a similar FWHM at each wavelength. We present the profiles of Pallas in the figures below for consistency, acknowledging the possibility that we have overestimated the size of the PSF at the longer wavelengths.

The FORCAST pipeline coadds the merged images. We use the standard deviation of the mean of fluxes extracted from the merged images (prior to coadding) as the $1 \sigma$ uncertainty of the fluxes in the coadded images of each of our targets. This uncertainty is negligible compared to the $6 \%$ uncertainty that we adopt for the flux calibration, per the GI Handbook Section 4.1 (Herter et al. 2013). The bandpasses of the selected FORCAST filters are such that only small color corrections are required. Based on the $F_{\nu} \propto \nu^{2}$ spectral shapes of our targets in the relevant ranges, we have applied color corrections of 1.004, $1.071,1.004,1.044,1.025$, and 1.025 to fluxes extracted from the F111, F197, F253, F315, F348, and F371 images, respectively. Aperture photometry was performed using the open-source Astropy (Astropy Collaboration et al. 2013) affiliated photutils ${ }^{8}$ package. Apertures span between $15^{\prime \prime}$ and $20^{\prime \prime}$, chosen to encompass the extended emission around each object. FORCAST photometry is reported in Table 2 and included in the SEDs in Section 3. Photometric error is reported as measured uncertainty in the sky background apertures.

\subsection{Adaptive Optics Mid-IR Imaging $(8-10 \mu \mathrm{m})$}

NML Cyg, S Per, and T Per were observed with the mid-IR adaptive optics system on the MMT using the Mid-infrared Array Camera and Bracewell Infrared Nulling Cryostat (MIRAC3/4/MIRAC-BLINC; Hoffmann et al. 1998; Hinz et al. 2000; Skemer et al. 2008). The observations of NML Cyg are described in Schuster et al. (2009), and discussed here in Section 3.5. S Per was observed on UT 2006 November 05 and T Per on UT 2009 October 02 at 8.9 and $9.8 \mu \mathrm{m}$. MIRAC achieved Strehl-ratios close to 0.95 , providing diffractionlimited imaging and stable PSFs (e.g., Biller et al. 2005).

\footnotetext{
8 Photutils provides tools for detecting and measuring the photometry of astronomical sources. The software is still in development, with documentation available at https://photutils.readthedocs.io/.
} 
Table 2

New Mid-infrared Photometry

\begin{tabular}{|c|c|c|c|c|c|c|c|c|c|c|}
\hline Name & $\begin{array}{c}8.8 \mu \mathrm{m}^{\mathrm{a}} \\
(\mathrm{Jy})\end{array}$ & $\begin{array}{c}9.8 \mu \mathrm{m}^{\mathrm{a}} \\
(\mathrm{Jy})\end{array}$ & $\begin{array}{c}11.1 \mu \mathrm{m}^{\mathrm{b}} \\
(\mathrm{Jy})\end{array}$ & $\begin{array}{c}19.7 \mu \mathrm{m}^{\mathrm{b}} \\
(\mathrm{Jy})\end{array}$ & $\begin{array}{c}25.3 \mu \mathrm{m}^{\mathrm{b}} \\
(\mathrm{Jy})\end{array}$ & $\begin{array}{c}31.5 \mu \mathrm{m}^{\mathrm{b}} \\
\text { (Jy) }\end{array}$ & $\begin{array}{c}34.8 \mu \mathrm{m}^{\mathrm{b}} \\
(\mathrm{Jy})\end{array}$ & $\begin{array}{c}37.1 \mu \mathrm{m}^{\mathrm{b}} \\
\text { (Jy) }\end{array}$ & $\begin{array}{l}70 \mu \mathrm{m}^{\mathrm{c}} \\
(\mathrm{Jy})\end{array}$ & $\begin{array}{c}160 \mu \mathrm{m}^{\mathrm{c}} \\
(\mathrm{Jy})\end{array}$ \\
\hline VX Sgr & $\cdots$ & $\cdots$ & $3740 \pm 98$ & $2250 \pm 91$ & $1400 \pm 85$ & $1090 \pm 69$ & $835 \pm 75$ & $697 \pm 10$ & $153 \pm 32$ & $23 \pm 11$ \\
\hline S Per & $316 \pm 52$ & $340 \pm 48$ & $\cdots$ & $342 \pm 28$ & $187 \pm 24$ & $146 \pm 15$ & $109 \pm 9.4$ & $97 \pm 9.6$ & $21 \pm 4.1$ & $3 \pm 1.2$ \\
\hline RS Per & & $\cdots$ & $\ldots$ & $82 \pm 4.8$ & $\ldots$ & $21 \pm 2.0$ & $\ldots$ & $15 \pm 4.1$ & $\ldots$ & $\cdots$ \\
\hline T Per & $8.8 \pm 1.4$ & $11.4 \pm 3.1$ & $\ldots$ & $9.7 \pm 1.2$ & $\ldots$ & $7.2 \pm 2.9$ & $\ldots$ & $\ldots$ & $\ldots$ & $\ldots$ \\
\hline NML Cyg $^{\mathrm{d}}$ & $3735 \pm 63$ & $3780 \pm 160$ & $\cdots$ & $4868 \pm 41$ & $3930 \pm 328$ & $3626 \pm 110$ & $2849 \pm 123$ & $2809 \pm 287$ & $652 \pm 96$ & $116 \pm 30$ \\
\hline
\end{tabular}

$\omega$

Notes.

${ }^{\mathrm{a}} \mathrm{MMT} / \mathrm{MIRAC}$.

${ }^{\mathrm{b}}$ SOFIA/FORCAST

${ }^{\mathrm{c}}$ HERSCHEL/PACS

${ }^{\mathrm{d}}$ NML Cyg MIRAC photometry originally presented in Schuster et al. (2009). 
Table 3

DUSTY Model Parameters and Mass-loss Rates

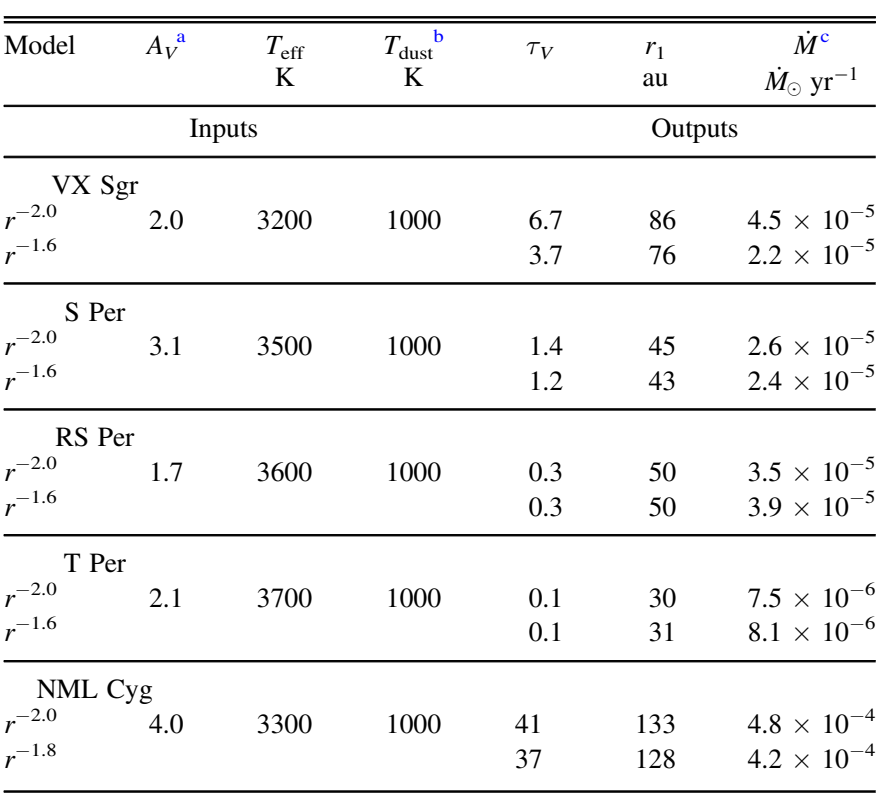

Notes.

${ }^{\text {a }}$ DUSTY output models are fit to extinction-corrected SEDs with these values of $A_{V}$.

${ }^{\mathrm{b}}$ Dust temperature at condensation radius, $r_{1}$.

c $\dot{M}$ is computed as an average mass-loss rate over the lifetime of the shell. The outflow velocity is assumed to be $25 \mathrm{~km} \mathrm{~s}^{-1}$ unless noted in the sections for the individual stars.

MIRAC4 employed a Si:As array with $256 \times 256$ pixels, and observations were made with a standard chop-and nod sequence to remove IR background emission. Cross-talk in the array electronics introduced faint artifacts in the horizontal and vertical directions, which is not completely removed by chop-and-nod subtraction. As described in Paper I, the horizontal cross-talk is mitigated during the data reduction with a code from Skemer et al. (2008). For consistency with the FORCAST photometry, we perform aperture photometry on the MIRAC images using photutils. We report the results in Table 2, include the photometry in the SEDs below, and as input to DUSTY.

\subsection{IRAS, AKARI, WISE, and ISO-SWS $(2-100 \mu \mathrm{m})$}

To populate the mid-IR SEDs, we include IRAS photometry (and $A K A R I$ photometry when available) from point-source catalogs in the literature for RS Per and VX Sgr (Smith et al. 2004), S Per and T Per (Abrahamyan et al. 2015), and NML Cyg (Schuster 2007). The Abrahamyan et al. (2015) catalog cross-correlates IRAS point sources with WISE, the latter of which presents some issues due to its large beam-size (up to $12^{\prime \prime}$ at $22 \mu \mathrm{m}$; Wright et al. 2010). For stars embedded in nebulosity or crowded fields, the WISE photometry can be systematically too bright.

Additionally, optical photometry is compiled from the Extended Hipparchos Compilation catalog (XHIP; Anderson \& Francis 2012), the SKY2000 Master Catalog (Myers et al. 2015), or the AAVSO Photometric All Sky Survey (APASS; Henden 2016, see SEDs in Section 3). These optical data, as well as the published photometry from IRAS and $A K A R I$, are dereddened using the extinction law from
O'Donnell (1994). The values for interstellar extinction $A_{V}$ chosen for each source are listed in Table 3 and in the SED captions below.

We also compile spectra from ISO-SWS (de Graauw et al. 1996) for all targets except T Per. S Per and RS Per spectra are from the Japanese guaranteed observing time program REDSTAR1 (PI T. Tsuji; Aoki et al. 1998), and NML Cyg and VX Sgr were observed with the AGBSTARS program (Justtanont et al. 1996; Speck et al. 2000). The color and extinction-corrected spectra are displayed in the SEDs below and are provided as near- to mid-IR photometric input to DUSTY.

\subsection{Herschel/PACS $(70,160 \mu \mathrm{m})$}

We also include in our analysis the publicly available 70 and $160 \mu \mathrm{m}$ observations made with Herschel/PACS. VX Sgr, NML Cyg, and S Per were observed as part of the Herschel key program Mass-loss of Evolved StarS (MESS; Groenewegen et al. 2011). The Herschel Interactive Processing Environment (HIPE; Ott et al. 2010) ${ }^{9}$ was used to download the images, but photometry was performed using photutils for consistency with the SOFIA images. Apertures span between 45 and $70^{\prime \prime}$ to encompass extended emission around each object. As the PACS pixels are large on sky, we did not have enough pixels in traditional sky annuli to model the background. Instead, we first mask the star and its nebulosity and then model the background across the field of view as a two-dimensional polynomial. For each of the PACS fields, these background models were fairly flat but had high rms variation. As summarized in Table 2, this uncertainty was as high as $\sim 40 \%$ for VX Sgr and S Per.

The width of the PACS bandpasses requires color corrections to be applied to the 70 and $160 \mu \mathrm{m}$ photometry from the images. In Paper I, the necessary corrections were estimated by convolving the "blue" $(70 \mu \mathrm{m})$ filter response functions to the sources' ISO LWS spectra. However, lacking spectra for all of the sources in this work, we instead fit the mid- to far-IR photometry from SOFIA and IRAS with a power-law of the form $F_{\nu}=\nu^{\beta}$ to represent the targets' SEDs at the PACS wavelengths. The results are modest corrections of 1.003 and 1.04 for the two bandpasses. PACS photometry is reported in Table 2 and included in the SEDs in Section 3. Photometric error is reported as measured uncertainty in the sky background models.

\section{Results and Discussion}

\subsection{DUSTY Modeling}

To estimate the mass-loss rates, mass-loss histories, and dust density distributions, we used the DUSTY radiative-transfer code (Ivezic et al. 1997) to model the observed SEDs and azimuthal average intensity profiles at each of the MIRAC and FORCAST wavelengths, in a manner similar to that used in Paper I. DUSTY solves the one-dimensional (1D) radiativetransfer equation for a spherically symmetric dust distribution around a central source. We provide as input the chosen optical properties, chemistry, size distribution of the dust grains, and a dust temperature, which fixes the inner boundary of the surrounding dust shell (the dust condensation radius, $r_{1}$ ). We

\footnotetext{
9 HIPE is a joint development by the Herschel Science Ground Segment Consortium, consisting of ESA; the NASA Herschel Science Center; and the HIFI, PACS, and SPIRE consortia.
} 


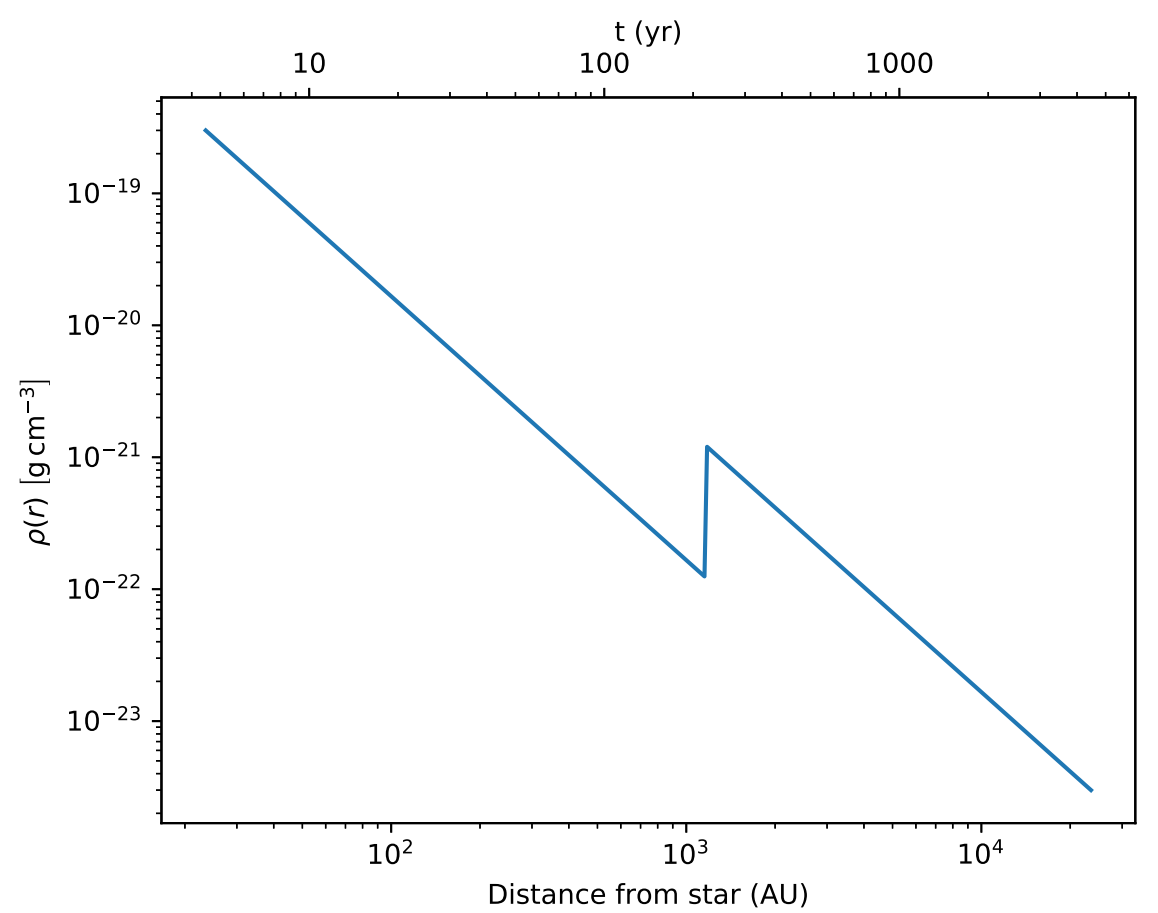

Figure 1. Example of an "enhanced" DUSTY model, where the dust density $\rho(r)$ follows an $r^{-2}$ distribution out to $\sim 50 \times r_{1}$ (where $r_{1}$ is the condensation radius corresponding to a dust condensation temperature of $1000 \mathrm{~K}$ ), at which point we simulate a discrete mass-loss event by enhancing the dust density distribution by a factor of 10 . The density units are artificially scaled in this example, but the distance at which the enhancement is placed for all of the models is roughly 1000 au from the central star. If we assume an outflow velocity of $25 \mathrm{~km} \mathrm{~s}^{-1}$, this distance corresponds to a high mass-loss event $\sim 200$ years ago.

generate a grid of models for each star with fixed stellar effective temperatures based on the spectral type of each target, fixed shell extent $\left(1000 \times r_{1}\right)$, and fixed dust condensation temperature $(1000 \mathrm{~K})$. Our grid consists of varying optical depths of the circumstellar material $\left(0.01<\tau_{V}<50\right)$ and different dust density distribution functions, described below. For a given set of inputs, DUSTY outputs a model SED and radial profiles of the dust shell at requested wavelengths.

As noted in Paper I, the spherical symmetry assumed by DUSTY fails to model the azimuthal complexities observed in the asymmetric outflows of massive stars such as VY CMa (Smith et al. 2001; Humphreys et al. 2005, 2007; Shenoy et al. 2013) and IRC +10420 (Humphreys et al. 1997; Tiffany et al. 2010; Shenoy et al. 2015). However, DUSTY allows for a consistent analysis of the dust, SEDs, and intensity profiles of the targets in this work and those in Paper I.

At a given wavelength, an output optical depth $\tau_{\lambda}$ from the model, and thus its grain opacity $\kappa_{\lambda}$, specifies the dust mass density $\rho(r)$ throughout the shell. If we assume a constant expansion rate $v_{\exp }$ of the outflowing dust shell, following the arguments set forth in Paper I, we can estimate the mass loss rate as:

$$
\dot{M}(t)=g_{d} 4 \pi r^{2} \rho(r) v_{\exp }
$$

where radius $r$ is a probe on timescale $t$ as $r=v_{\exp } t$, and $g_{d}$ is the gas-to-dust ratio. For consistency with Paper I, we assume $g_{d}=100: 1$ (Knapp et al. 1993); however, this can be as high as 200:1 for supergiants (Decin et al. 2006; Mauron \& Josselin 2011). For the dust optical properties, we use the "cool" circumstellar silicates from Ossenkopf et al. (1992), and assume the grain radii follow a Mathis, Rumpl, Nordsieck (MRN) size distribution $n(a) \propto a^{-3.5} d a$ (Mathis et al. 1977) with $a_{\min }=0.005 \mu \mathrm{m}$ and $a_{\max }=0.25 \mu \mathrm{m}$.
In general, the mass density distribution of the outflows can be modeled with DUSTY as a power-law $\rho(r) \propto r^{-q}$. An index of $q=2$ is the case of constant mass-loss rate and constant expansion velocity for the shell, while $q<2$ indicates a gradual decline in the mass-loss rate over the dynamical age of the expanding shell. A steeper power-law index $q>2$ represents a mass distribution with more recent high mass loss, and less significant mass loss in the past. For each of our targets, the fundamental research question is how well the stars' SEDs and radial profiles in the mid-IR can be modeled with a constant mass-loss rate scenario in DUSTY.

For each of the targets in our sample, we perform three Monte Carlo experiments. In the first, we force DUSTY to use the constant mass-loss rate distribution $\rho(r) \propto r^{-2}$, and by varying the optical depth of the CS material, recover the bestfitting, constant mass-loss SED in the near- to mid-infrared. For the second set of simulations, we allow the power-law index of the mass distribution to vary between 1 and 3 with a step size of 0.2 , deliberately excluding the $r^{-2}$ case, while also allowing the optical depth to vary. For both set of DUSTY models, we evaluate the best fit based on a reduced $\chi^{2}$ measurement of the extinction-corrected SED and the DUSTY output spectrum. We then compare the DUSTY-predicted intensity profiles to the observed radial profiles in the SOFIA wavelengths (and MIRAC, when available). The image and profile models output from DUSTY do not account for the optics of the telescopes, so the intensity profiles are convolved with an azimuthal average of the PSF and are displayed in the figures below.

For the third and final set of DUSTY models, we select the best-fitting $r^{-2}$ model, and re-run DUSTY with those same parameters, this time enhancing the dusty density profile by a factor of 10 at 50 condensation radii $\left(50 \times r_{1}\right)$. An example model is shown in Figure 1. These "enhanced," piecewisedefined models explore the possibility of an extreme mass-loss 


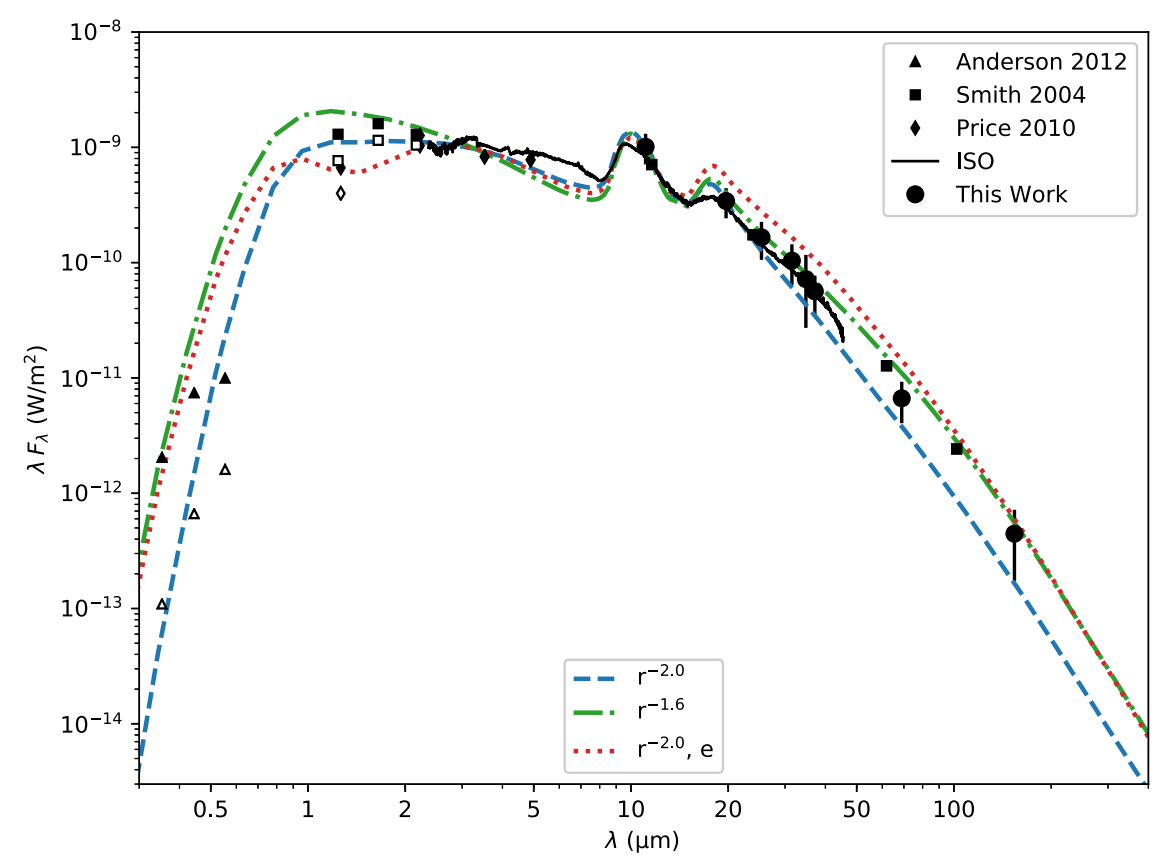



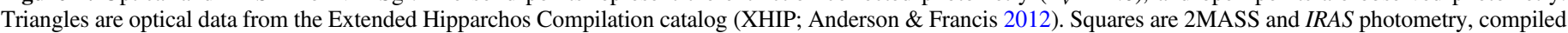

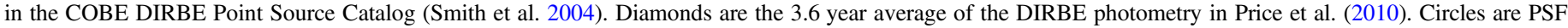

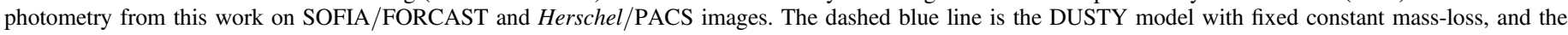

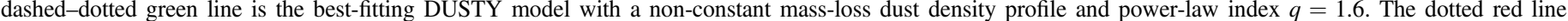



event in a star's past, similar in some respects to the models from the second experiment described above where the density distribution can be shallower than $r^{2}$. The latter DUSTY models imply a smoothly changing mass-loss rate over the lifetime of the star, whereas the "enhanced" models simulate a single eruptive event in the mass-loss history. Similar piecewise defined density profiles were used in Paper I to model the SED of IRC +10420 . Though a factor of 10 enhancement in mass-loss rate is likely an extreme case, we apply this model to explore how well the scenario of a constant mass-loss rate with a single one-off eruptive event reproduces the observed IR SED of our target stars.

Finally, we can estimate an average mass-loss rate for the non-constant mass-loss models $(q \neq 2)$ by integrating the density distribution $\rho(r)$ and multiplying by the gas-to-dust mass ratio (100:1) to compute the total mass of the shell $M$. We assume an average expansion velocity to estimate the dynamical age of the shell $\Delta t=r_{2} / v_{\exp }$ where $r_{2}$ is the outer radius of the shell predicted by a given model. The expansion velocity, $v_{\text {exp }}$, is assumed to be $25 \mathrm{~km} \mathrm{~s}^{-1}$ unless specified in the sections for individual stars below. The average mass-loss rate is then $\langle\dot{M}\rangle=M / \Delta t$. The specific parameters for the DUSTY models for each target in our program, as well as the output DUSTY models and computed average mass-loss rates, are summarized in Table 3, and the best-fitting SEDs to the observed photometry are shown in the figures below.

Note that in Table 3, the first row for each target star represents the best-fitting DUSTY simulations forced to evaluate the models in the constant mass-losing, $r^{-2}$ dust profile case. The second row represents the best-fitting SED with non-constant mass-loss. The columns on the left reflect the input values, and the right-hand columns are the recovered output parameters from the best-fitting models for each target and each simulation set (constant versus non-constant mass-loss rates). We do not include the enhanced, piecewisedefined models here, as the parameters were fixed to the $r^{-2}$ model for each star. Throughout the text, we will refer to the three different models as constant $\left(r^{-2}\right)$, non-constant $\left(r^{-q}, q \neq 2\right)$, and enhanced $\left(r^{-2}, e\right)$ mass-loss rates.

\section{2. $V X S g r$}

VX Sgr has a marginally resolved, nearly symmetric extended circumstellar envelope in its HST visual images (Schuster et al. 2006). Additionally, Vlemmings et al. (2005) has identified a dipole magnetic field in its ejecta mapped by its $\mathrm{H}_{2} \mathrm{O}$ masers, which may be a clue to its mass loss mechanism. VX Sgr is also a semi-regular variable that behaves like a fundamental mode pulsator (i.e., a Mira variable), which is rare for such a luminous star. It has been observed to vary by several magnitudes with corresponding changes in its apparent spectral type from M4 to M10.

During one of its Mira-like episodes, Humphreys \& Lockwood (1972) noted a decline of $\sim 0.5$ mag out to $10 \mu \mathrm{m}$ over a few months. Therefore, we have chosen optical photometry to align in light-curve phase with the 2MASS and IRAC photometry compiled in Smith et al. (2004). To constrain the $2-10 \mu \mathrm{m}$ regime of the SED, we also include the photometric average over the 3.6-year cycle of the COBE DIRBE project (Price et al. 2010).

The SED is shown in Figure 2, with observed data plotted as open symbols and extinction-corrected photometry in solid. The constant mass-loss DUSTY model is overplotted with a dashed blue line, and the best-fitting power-law model with index $q=1.6$ is shown by the dashed-dotted green line. Note that both models fit the $10 \mu \mathrm{m}$ silicate feature in the ISO spectrum, but the $q=1.6$ model better simulates the cool thermal dust emission out to $100 \mu \mathrm{m}$. However, the observed 


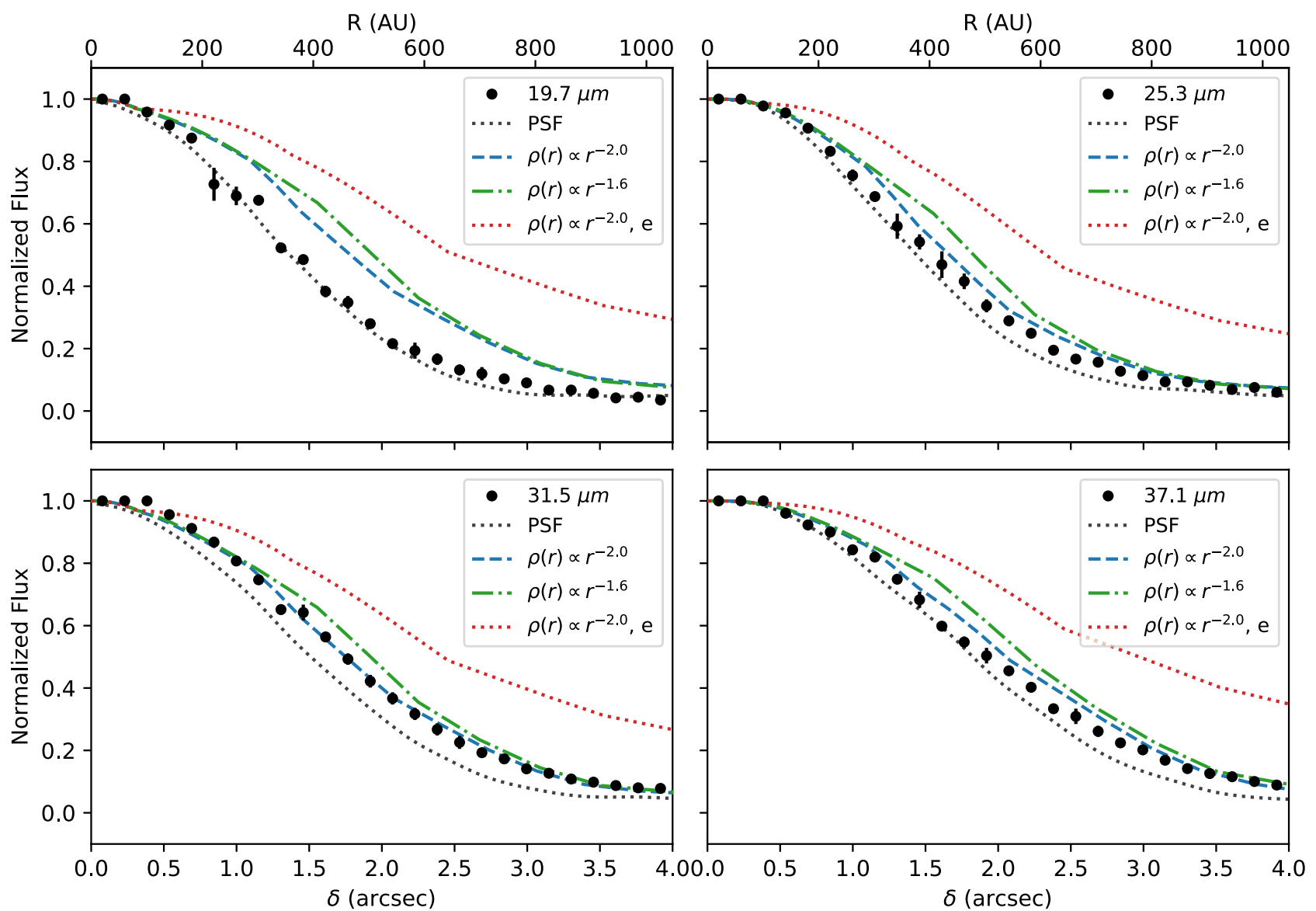

Figure 3. Radial profiles of VX Sgr at four SOFIA wavelengths (black dots). The dotted gray line shows the PSF measured at each wavelength from images of the asteroid Pallas. The dashed blue line represents the modeled profile from DUSTY assuming a constant mass-loss rate, which implies a dust density distribution of $\rho(r) \propto r^{-2}$. The dashed-dotted green line is the best-fitting model to the SED from DUSTY, excluding the non-constant case, and the dotted red line is the "enhanced" DUSTY model. All models are convolved with the Pallas PSF. We note that the circumstellar ejecta around VX Sgr is only marginally resolved above the FORCAST PSF at $19.7 \mu \mathrm{m}$, and all three models over-estimate the amount of dust emission expected at the FORCAST wavelengths.

IR flux from $\sim 30$ to $70 \mu \mathrm{m}$ falls in between the two models. Displayed in dotted red is the "enhanced" DUSTY model, which is the $r^{-2.0}$ constant mass-loss model with a factor of 10 enhancement in dust density at $50 \times r_{1}$. This model appears to over-estimate the thermal dust emission, implying too much dust is produced to match the observations of VX Sgr.

The derived mass-loss rates for the models are summarized in Table 3. The outflow velocity $v_{\exp }$ adopted for this calculation is $24.3 \mathrm{~km} \mathrm{~s}^{-1}$ from the AGB/supergiant CO-line survey by De Beck et al. (2010). The mass-loss rates from DUSTY $\left(2-5 \times 10^{-5} M_{\odot} \mathrm{yr}^{-1}\right)$ are somewhat lower than the measurements from CO-line profiles $\left(6.1 \times 10^{-5} M_{\odot} \mathrm{yr}^{-1}\right.$; De Beck et al. 2010). However, the most obvious explanation for this is due to the assumed gas-to-dust ratio. Where we have assumed 100:1 for consistency with Paper I, De Beck et al. (2010) allow the gas-to-dust ratio to vary when fitting the observed outflow velocities (using GASTRoNOoM; Decin et al. 2006). Mass-loss rates scale linearly with the gas-to-dust ratio, so if we had applied a ratio of 200:1, perhaps more appropriate for RSGs (Decin et al. 2006; Mauron \& Josselin 2011), our estimated mass-loss rate would be more consistent with the derived measurement from CO-line profiles.

In Figure 3, we compare the observed radial profiles to the PSF calibrator (2 Pallas) and the DUSTY output image models. The ejecta around VX Sgr is only marginally resolved above the PSF at $19.7 \mu \mathrm{m}$, but the envelope is more easily distinguished from the PSF at longer wavelengths. The DUSTY model profiles are convolved with the PSF in each band, and we note that the constant mass-loss rate model aligns more closely with the observed surface brightness profiles of VX Sgr. Both the shallower $r^{-1.6}$ and enhanced models overestimate the amount of thermal dust emission observed in the FORCAST images.

The azimuthal profiles combined with the SED modeling suggest that the mass-loss rate of VX Sgr is fairly constant with perhaps a period of elevated mass-loss in the past, as illustrated by the infrared excess emission in the $20-160 \mu$ m photometry. The model intensity profiles, though, all predict a higher surface brightness for the extended emission than what was actually observed at SOFIA wavelengths (Figure 3). One possible explanation for not observing this emission is that VX Sgr has experienced a sudden decline in mass-loss rate in very recent times. Exploring this possibility is beyond the scope of this paper, but we plan to make high-resolution 5-12 $\mu \mathrm{m}$ observations using LMIRCam and NOMIC on the LBT (Skrutskie et al. 2010; Hoffmann et al. 2014). At FWHM spatial resolutions of $0 . " 12$ and 0.29 at 5 and $12 \mu \mathrm{m}$, respectively, we can explore the dust shell at $\sim 200$ au scales and combine these observations with our SOFIA data and DUSTY modeling. 


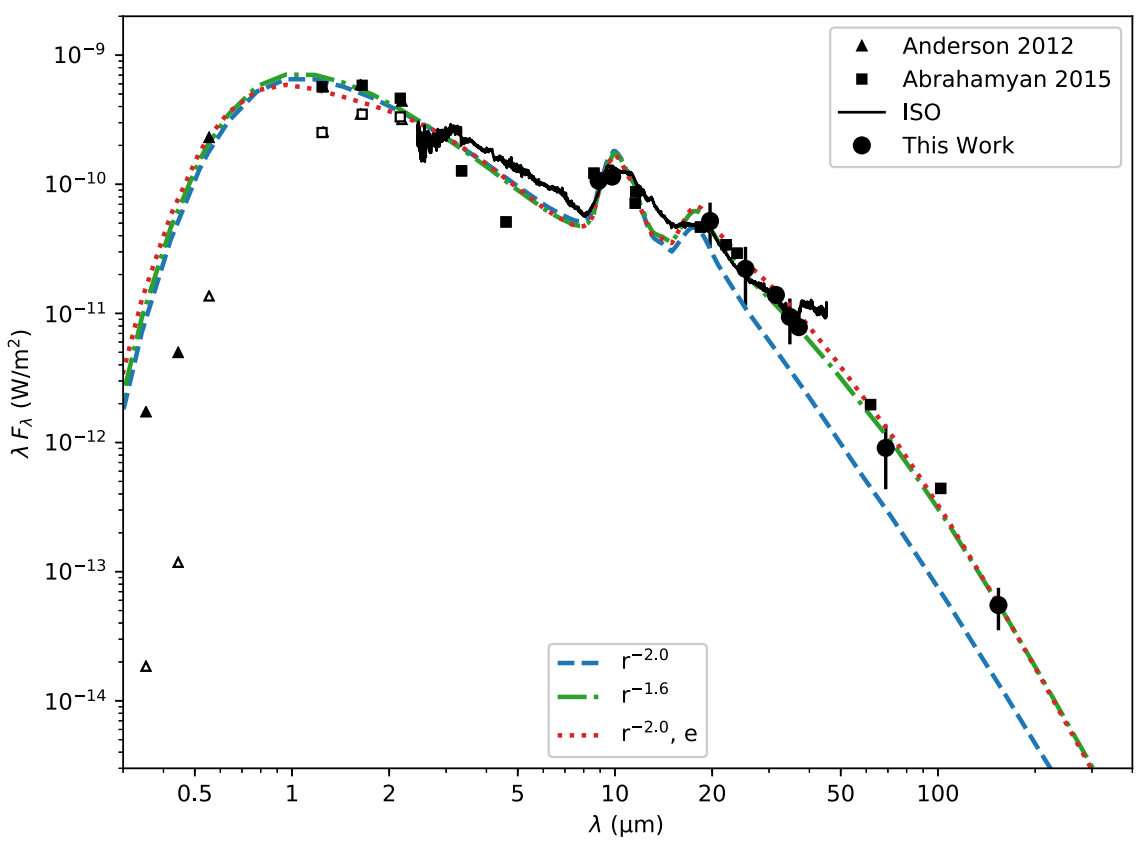

Figure 4. Optical and IR SED of S Per. The solid points represent the extinction-corrected photometry $\left(A_{V}=3.1\right)$, and open points are observed photometry. Triangles are optical data from the Extended Hipparchos Compilation catalog (XHIP; Anderson \& Francis 2012). Squares are from the 2MASS, WISE, IRAS, and AKARI point-source catalogs, compiled in Abrahamyan et al. (2015). Circles are PSF photometry from this work on SOFIA/FORCAST and Herschel/PACS images. The dashed-dotted green line is the best-fitting model to the SED from DUSTY, excluding the non-constant case, and the red dotted line is the "enhanced" model.

We also note that although the envelope is spherically symmetric in HST optical images (Schuster et al. 2006), the $\mathrm{H}_{2} \mathrm{O}$ masers around VX Sgr appear to align with the equatorial plane of the star's dipole magnetic field. Vlemmings et al. (2005) suggest that this alignment could create an overdensity in the circumstellar material in this plane, as modeled by Matt et al. (2000). While we do not see evidence for asymmetry in the FORCAST images, we note again that DUSTY assumes spherical symmetry in its models. As discussed further with S Per and NML Cyg below, it is likely that DUSTY may fail to accurately model stars with known asymmetric outflows and profiles.

\section{3. $S$ Per}

S Per (Sp. Type M3-4e Ia) is an OH/IR source and a member of the Per OB1 association (Humphreys 1978), with a distance of $2.3 \pm 0.1 \mathrm{kpc}$ as determined by VLBI $\mathrm{H}_{2} \mathrm{O}$ maser astrometry (Asaki et al. 2010). Schuster et al. (2006) present $H S T$ images showing that the star is embedded in an elongated circumstellar envelope with a position angle of $\sim 20^{\circ} \mathrm{E}$ of $\mathrm{N}$ with a FWHM of $\sim 0$ !" 1 (240 au). Schuster et al. (2006) speculated that the shape could be due to bipolarity in the star's ejecta or a flattened circumstellar halo, and they note this elongated structure is also seen in $\mathrm{OH}$ and $\mathrm{H}_{2} \mathrm{O}$ maser observations on the same scale and with similar orientation (Richards et al. 1999; Vlemmings et al. 2001). Fitting elliptical Gaussians to S Per's MIRAC4 images yields a mean position angle of $19^{\circ} \pm 2^{\circ} \mathrm{E}$ of $\mathrm{N}$, matching the orientation seen in the HST images.

The observed SED is shown in Figure 4 along with the three DUSTY models. Both the shallower $q=1.6$ and enhanced DUSTY models accurately reconstruct the near- to mid-IR flux, while the constant mass-loss rate model underestimates the thermal dust emission. We note here one possible complication in our analysis. DUSTY simulations assume spherical symmetry in CS material, which could lead to underestimating the density, and thus optical depth, of the ejecta relative to the observed compact envelope seen in the Schuster et al. (2006) WFPC2 images of S Per. Our models for this star, then, may not best represent the stellar outflows and dusty envelope.

The observed azimuthal average radial profiles from SOFIA/FORCAST for S Per are presented in Figure 5. S Per has resolvable extended emission above the PSF; however, the $q=2$ and $q=1.6$ profiles, once convolved with the large PSF beam of FORCAST, are virtually indistinguishable. The enhanced DUSTY model, though, predicts too much emission close to the central star.

In Figure 6, we illustrate the surface brightness profiles at higher spatial resolution with MIRAC. Here, the observed surface brightness profile is clearly resolved above the PSF at the shorter wavelengths; however, the DUSTY models underestimate the shape of the stellar envelope. Adding a period of enhanced mass loss, the DUSTY model in dotted red, produces too much emission at the shorter wavelengths. Unfortunately, then, the radial profile models do not provide any conclusive evidence that the mass-loss history of S Per is constant versus non-constant. Note that the deviations from a smooth profile in the $31.5 \mu \mathrm{m}$ and the two MIRAC figures are due to the asymmetry in the outflows. From the SED, though, we glean that S Per may have had a higher mass-loss rate in the past, but we acknowledge that DUSTY is not ideal for simulating stellar ejecta of stars with known bipolar/asymmetric envelopes.

As reported in Table 3, the two DUSTY models predict mass-loss rates between $\sim 2$ and $3 \times 10^{-5} M_{\odot} \mathrm{yr}^{-1}$. Richards et al. (1999) summarizes results from previous literature to show a range of published mass-loss rates from as low as $7 \times 10^{-6} M_{\odot} \mathrm{yr}^{-1}(\mathrm{OH} 1612 \mathrm{MHz}$; Jura \& Kleinmann 1990) to as high as $2 \times 10^{-4} M_{\odot} \mathrm{yr}^{-1}$ (CO-line profiles; Knapp \& Morris 1985). With such a large range of published values, each measuring mass-loss rates with a different observational 

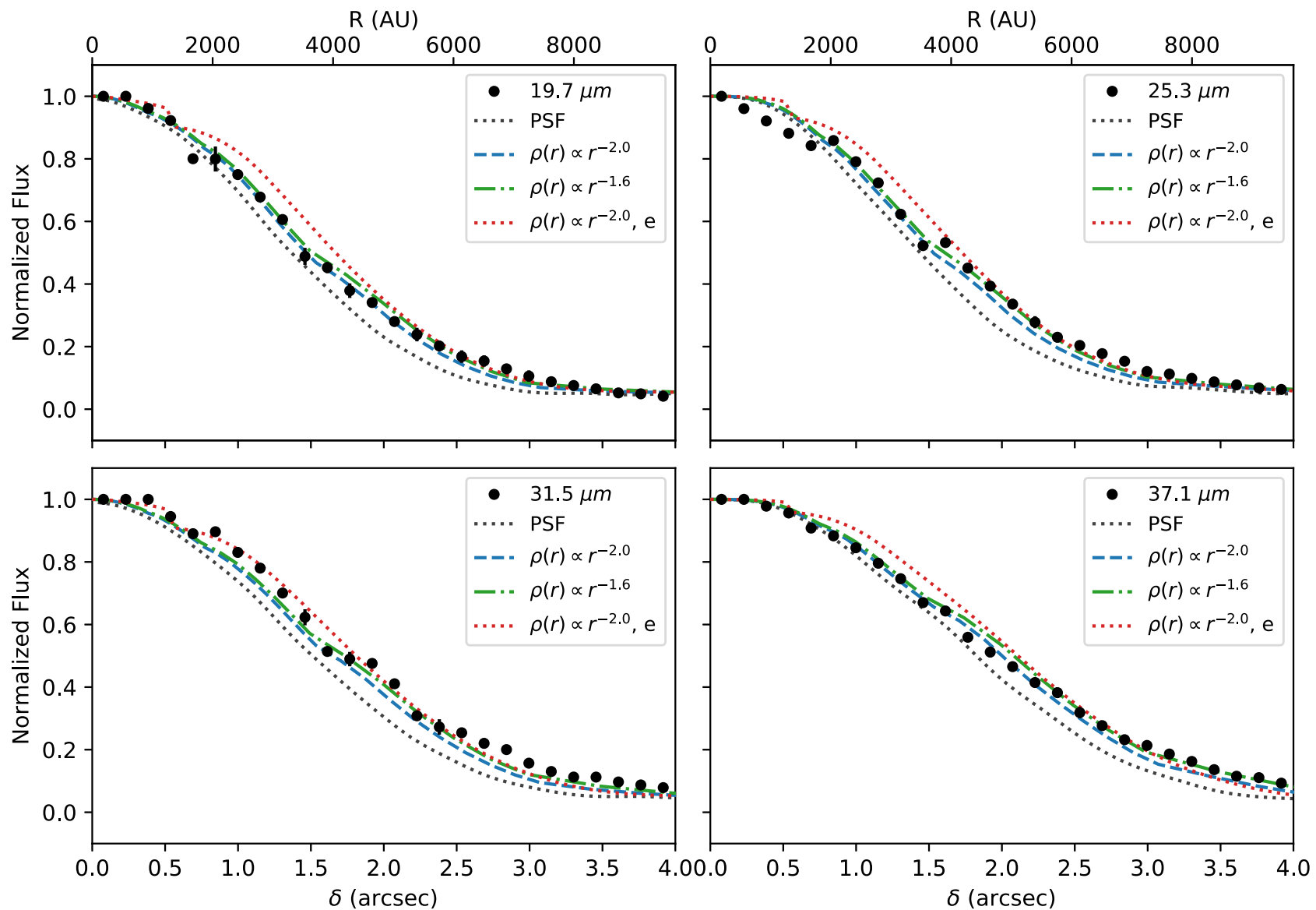

Figure 5. Radial profiles of S Per at the SOFIA/FORCAST wavelengths, similar to Figure 3. S Per has resolvable extended envelope emission above the PSF flux; however, the predicted profiles from DUSTY are too similar in shape to distinguish one over the other as a best-fitting model to the observed surface brightness profile. Still, the enhanced DUSTY model perhaps over-predicts the amount of thermal emission that would be observed close in to the central star.


Figure 6. Radial profiles of S Per from the two MIRAC filters. The observed profile shape has bumps and ridges due to the asymmetry of the envelope in the NE-SW direction. The PSF shown is of $\beta$ And.

technique, we can only conclude that we have derived a rate within published bounds.

Fok et al. (2012) also performed DUSTY modeling on a number of Galactic RSGs, including S Per, T Per, and RS Per. However, they used a different mode, the "dusty AGB" radiatively driven wind mode, and a higher gas-to-dust ratio of 200:1. The radiatively driven wind mode in DUSTY is provided for modeling AGB star envelopes and is not necessarily appropriate for RSGs (e.g., Heras \& Hony 2005). Groenewegen (2012) analyzed the systematic difference in mass-loss rates computed using DUSTY in this mode as compared to the default where the user supplies the density distribution as a power-law function. Groenewegen (2012) found that the mass-loss rates computed with the radiatively 


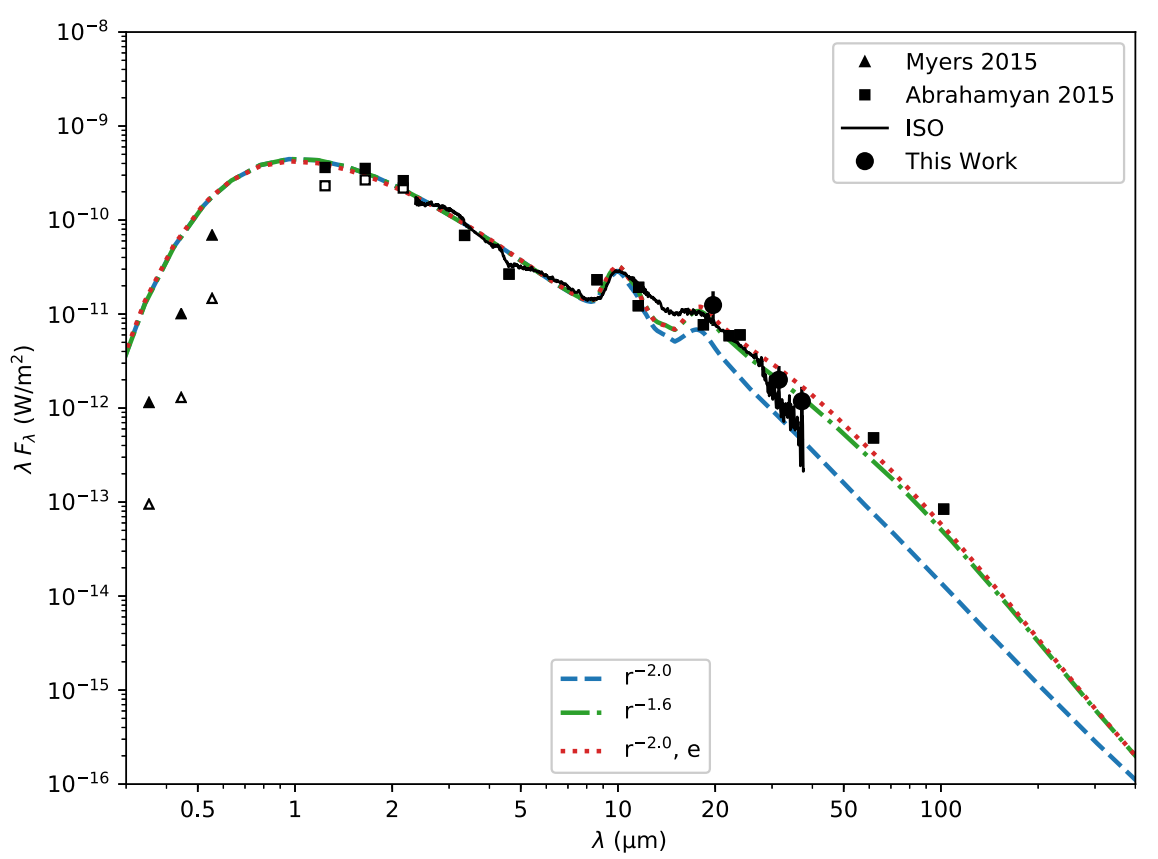

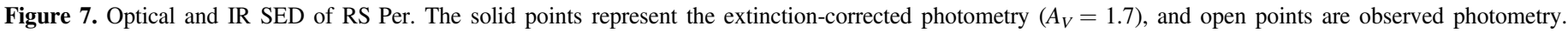

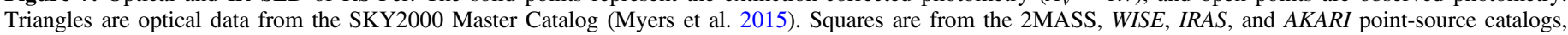
compiled in Abrahamyan et al. (2015). Circles are PSF photometry from this work on SOFIA/FORCAST images.

driven wind mode differ significantly from those obtained from the default mode in which the equation of radiative transfer alone is solved when applied in the context of RSGs. Thus, our results, with fixed single-component power-law distributions, are not directly comparable to the Fok et al. (2012) models. Nonetheless, Fok et al. (2012) yield a best-fitting model with $\dot{M}=1 \times 10^{-5} M_{\odot} \mathrm{yr}^{-1}$. We note that Gehrz \& Woolf (1971) derived a mass-loss rate for S Per of $2.7 \times 10^{-5} M_{\odot} \mathrm{yr}^{-1}$ using an independent analysis of the 3.6-11.4 $\mu \mathrm{m}$ SED. These results are consistent with our measurements.

\subsection{RS Per and T Per}

Departures from circular symmetry have been reported for both RS Per and T Per based on H-band interferometric imaging with CHARA by Baron et al. (2014) at an angular scale of 1.3 mas, which the authors attribute to surface asymmetries or spots. We do not see much evidence for asymmetry in the FORCAST images, and the azimuthal profiles for RS Per do not show significant excess emission above the PSF, though the angular scales for FORCAST are much larger than Baron et al. (2014) observed with the CHARA array. RS Per has the $10 \mu \mathrm{m}$ silicate emission feature in its SED but is not a known maser source. It is likely a normal RSG that may just be entering a more active phase with enhanced mass loss, perhaps driven by surface activity like that seen in the $\mathrm{OH} / \mathrm{IR}$ supergiants and VY CMa. T Per, another member of the Perseus OB1 association, similarly shows no evidence for $\mathrm{SiO}$ maser emission (Jiang et al. 1999). Both stars exhibit long-period variability of $\sim 4200$ and 2500 days for RS Per and T Per, respectively (AAVSO; Kiss et al. 2006).

The SED of RS Per is shown in Figure 7. Both models fit the $10 \mu \mathrm{m}$ silicate feature, as well as the ISO spectrum, from 2-11 $\mu \mathrm{m}$. However, the constant mass-loss, $q=2$ profile underestimates the flux for the mid- to far-IR at wavelengths larger than $20 \mu \mathrm{m}$, while the shallower dust density distribution $q=1.6$ profile and the enhanced $r^{-2.0}$ profile better match the longer wavelength SED. However, after convolution with the FORCAST PSF, the radial profiles of both models in Figure 8 appear too similar to the constant mass-loss case beyond 2" to distinguish either as a better fit to the extended emission. For T Per, shown in Figure 9, both the constant mass-loss and enhanced models produce insufficient thermal dust emission at the longer wavelengths.

As summarized in Table 3, the mass-loss rates derived for RS Per and T Per are $4 \times 10^{-5}$ and $8 \times 10^{-6} M_{\odot} \mathrm{yr}^{-1}$, respectively. Fok et al. (2012) estimates a mass-loss rate of $3.0 \times 10^{-6} M_{\odot} \mathrm{yr}^{-1}$ for RS Per and $5 \times 10^{-7} M_{\odot} \mathrm{yr}^{-1}$ for T Per using DUSTY "AGB mode." As discussed in Section 3.3, this radiatively driven wind mode is less appropriate for RSGs, and so our results are not directly comparable. Additionally, we note that the SED fits provided in their work do not extend longward of $30 \mu \mathrm{m}$, so we cannot qualitatively gauge which SED modeling mode (our power-law profiles versus their radiatively driven wind models) would fit best with our new FORCAST photometry through $40 \mu \mathrm{m}$. Particularly in the case of $\mathrm{T}$ Per, we note that this longwavelength IR photometry is crucial in constraining the models.

While the $r^{-1.6}$ DUSTY model is clearly the better fit to the observed mid-IR SED, the radial profiles of RS Per in Figure 8 reveal that the SOFIA images lack the spatial resolution necessary to favor one model over the other as a better fit to the extended envelope emission. The radial profiles of $\mathrm{T}$ Per in Figures 10 and 11 reveal a much clearer extended profile above the PSF at $31.5 \mu \mathrm{m}$ and around the $10 \mu \mathrm{m}$ silicate feature with MIRAC. However, the two power-law models predict very similar output profiles. The enhanced model accurately recovers the extended emission around the $10 \mu \mathrm{m}$ silicate feature, but the MIRAC images in the two other wavebands do not resolve emission extended above the PSF.

We note a curious ripple in the $31.5 \mu \mathrm{m}$ FORCAST profile in Figure 10. As mentioned in Section 2.1, some optical 

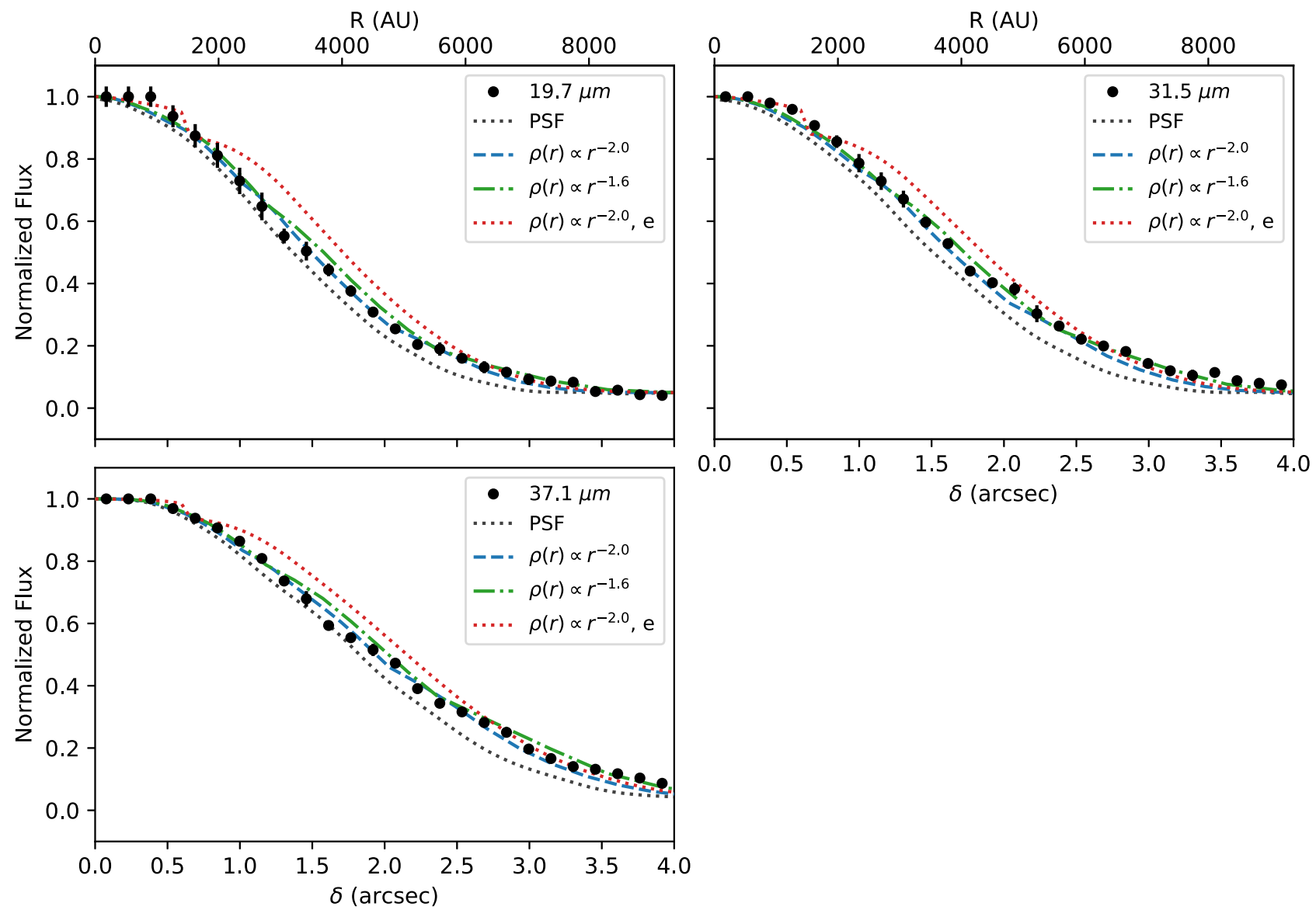

Figure 8. Radial profiles of RS Per at the SOFIA/FORCAST wavelengths, similar to Figure 3. The figures reveal extended emission above the PSF at 2-4" from the central star. The shallower mass-loss model $\left(\rho(r) \propto r^{-1.6}\right)$, though a better fit to the photometry in Figure 7, appears too similar to the constant mass-loss model after convolution with the PSF to conclude which mass-loss history is a better fit.



Figure 9. Optical and IR SED of T Per. The solid points represent the extinction-corrected photometry $\left(A_{V}=2.1\right)$, and open points are observed photometry. Triangles are optical data from the AAVSO Photometric All Sky Survey (APASS; Henden 2016). Squares are from the 2MASS, WISE, IRAS, and AKARI point-source catalogs, compiled in Abrahamyan et al. (2015). Diamonds are MSX photometry in Egan et al. (2003). Circles are PSF photometry from this work on SOFIA/ FORCAST and MMT/MIRAC images. 


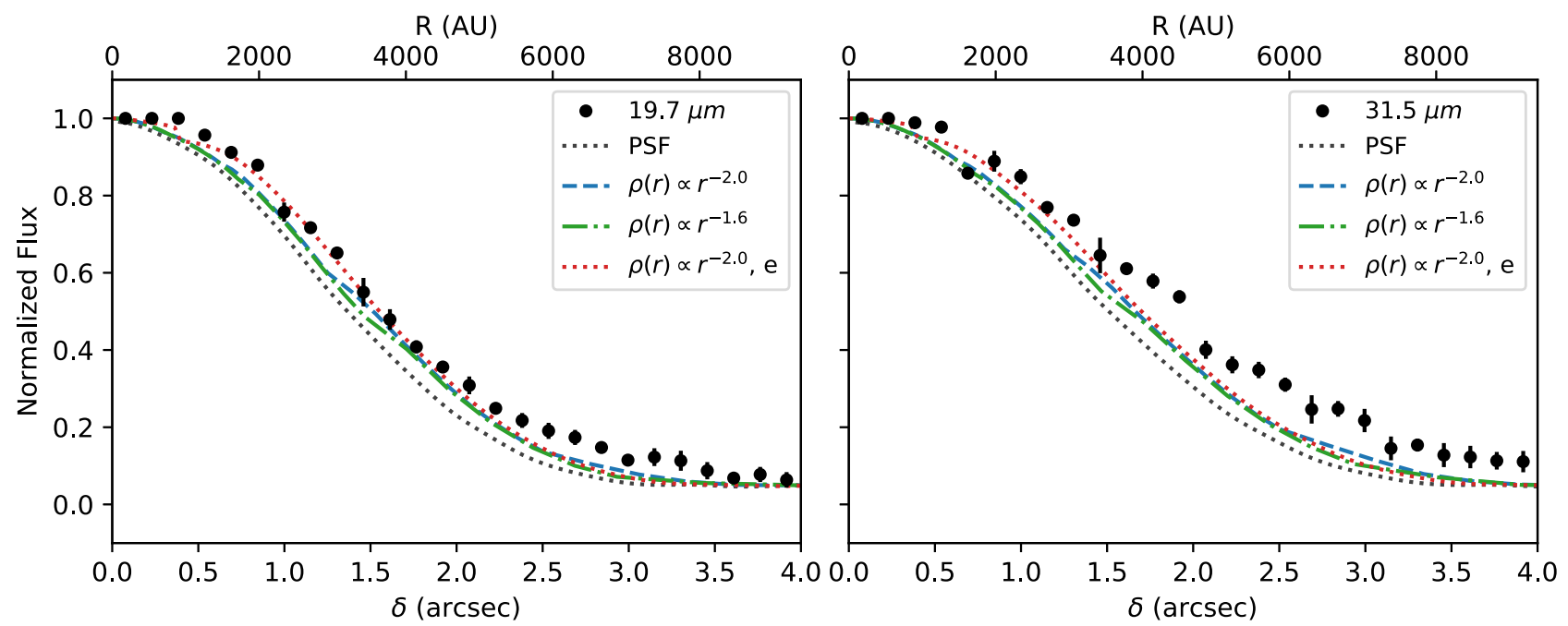

Figure 10. Radial profiles of T Per at the SOFIA/FORCAST wavelengths, similar to Figure 3. T Per has obvious extended emission above the PSF at $31.5 \mu$ m, though neither model reproduces this observed profile. The shape of the observed radial profile suggests a geometry more complicated than a single power-law distribution can generate. T Per may have undergone multiple eruptive mass-loss events, the outflows are asymmetric, or we are seeing coma effects in our images (see discussion in Section 3.4).
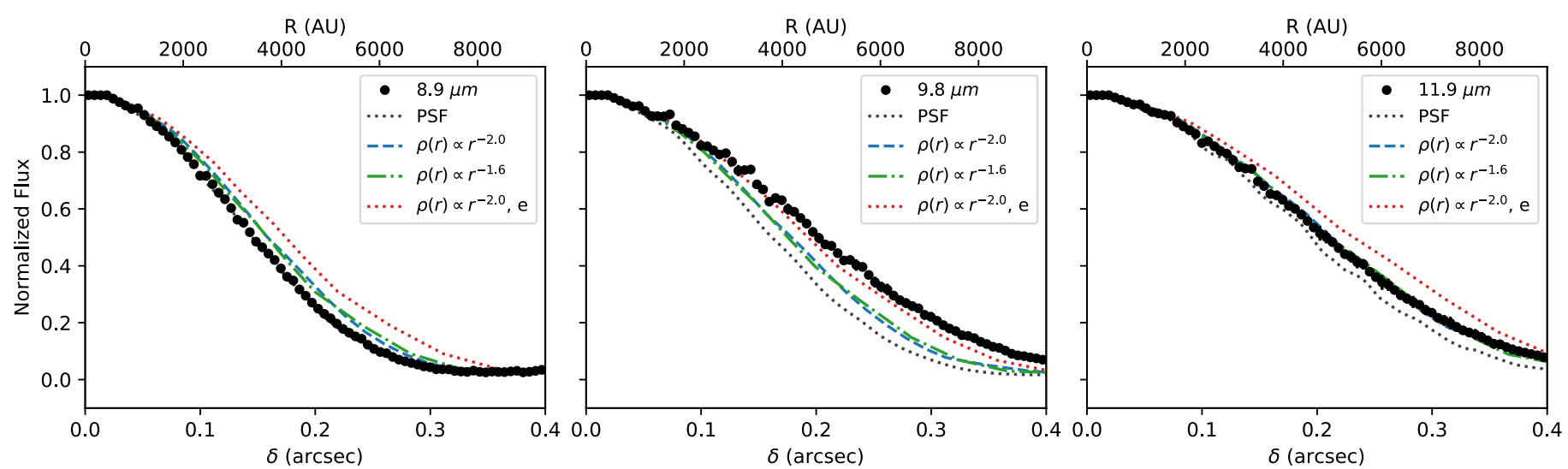

Figure 11. Radial profiles of T Per from the three MIRAC filters. T Per has clearly resolved extended emission above the PSF ( $\beta$ And) at $\lambda>9.8 \mu$ m. Although the shallower $r^{-1.6}$ model is a better fit at the longer SOFIA wavelengths (Figure 10), the enhanced mass-loss rate DUSTY model reproduces the shape of the observed profile at $\sim 10 \mu \mathrm{m}$ in the MIRAC images.

aberrations may be introduced to the images due to chopping patterns of the secondary mirror on SOFIA. One possible source of the profile shape could be coma effects that stretch out $\mathrm{T}$ Per along one axis. When generating azimuthally averaged surface brightness profiles, this asymmetry would cause the profile to deviate from a smooth power-law.

\subsection{NML Cyg}

$H S T$ visual images of NML Cyg revealed a peculiar beanshaped asymmetric nebula only $\approx 0$ !' 2 across and coincident with the distribution of its $\mathrm{H}_{2} \mathrm{O}$ masers. Schuster et al. (2006) showed that its circumstellar envelope is shaped by photodissociation from the powerful nearby association Cyg OB2 inside the Cygnus X superbubble, which is relatively void of gas and dust. This configuration allows the UV radiation from the numerous luminous hot stars in Cyg OB2 to travel the $\approx 80 \mathrm{pc}$ to NML Cyg unimpeded. Subsequent adaptive optics mid-IR imaging at 8.8, 9.8, and $11.7 \mu \mathrm{m}$ with MIRAC3 on the MMT (Schuster et al. 2009) spatially resolve the physical structures near the star $(\sim 240 \mathrm{au})$ responsible for its $10 \mu \mathrm{m}$ silicate-absorption feature and an asymmetric excess at
0 ". $3-0$." 5 from the star due to thermal emission from hot dust. This excess is also oriented toward the Cyg OB2 association and is attributed to the destruction of NML Cyg's dusty wind by the hot stars in Cyg OB2.

As illustrated in the SED in Figure 12, the $10 \mu \mathrm{m}$ silicate feature is seen in absorption, rather than emission. Discussed in detail in Schuster et al. $(2006,2009)$, this absorption is due to NML Cyg's thick circumstellar envelope obscuring the central star. Indeed, the DUSTY models predict a large optical depth $\left(\tau_{V}>40\right.$ for both models) to fit the silicate feature in absorption as well as the mid- to far-IR photometry.

At the FORCAST wavelengths, 19.7-37.1 $\mu \mathrm{m}$, NML Cyg appears as a point source, with no evidence of cold dust hidden or protected from the UV radiation in Cyg OB2. Additionally, no preferential extension toward Cyg OB2 is evident at the angular resolution of FORCAST. The radial profiles in Figure 13 do not show any obvious excess emission above the PSF. While we note that the shape of the observed azimuthal profile seems similar to the $r^{-2}$ model, the models seem to greatly over-predict the surface brightness flux for the large estimated optical depth. 


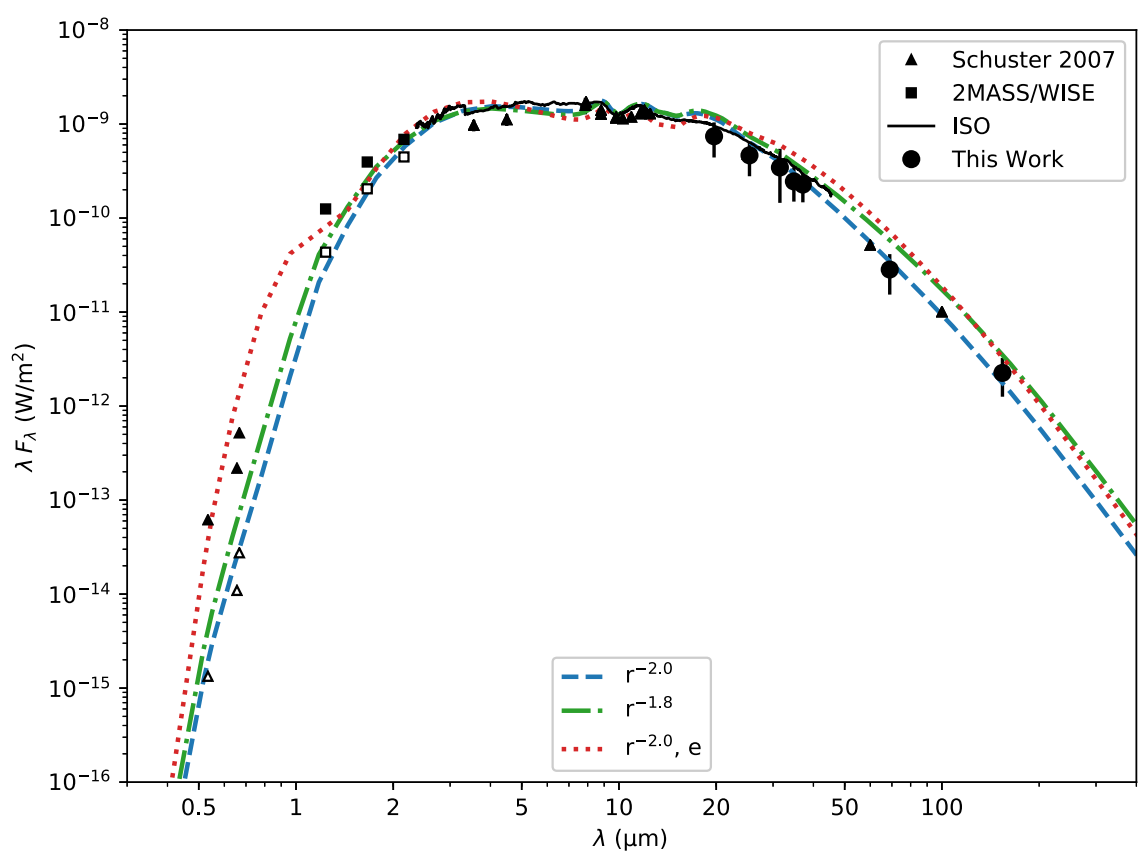

Figure 12. Optical and IR SED of NML Cyg. The solid points represent the extinction-corrected photometry $\left(A_{V}=4.0\right.$; see Schuster 2007), and open points are observed photometry. Triangles are from observations in Schuster (2007) using $H S T /$ WFPC2 at $V, H_{\alpha}$, and $R$; MMT/MIRAC3 at 9-12 $\mu$ m; the OBO Bolometer at 1-12 $\mu \mathrm{m}$; and Spitzer/IRAC and IRAS photometry. Squares are from the 2MASS and WISE point-source catalogs. Circles are PSF photometry from this work on SOFIA/FORCAST and Herschel/PACS images.
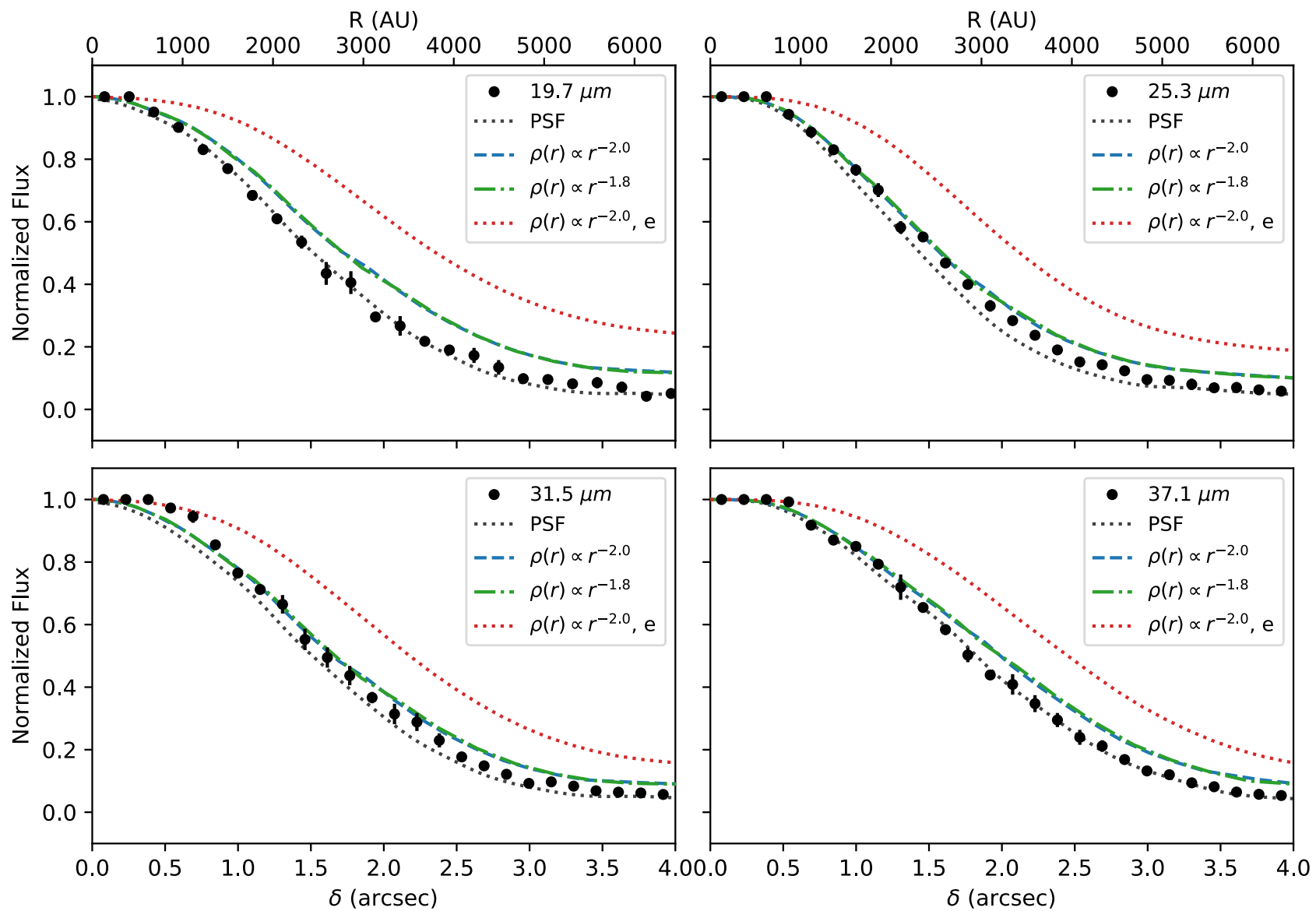

Figure 13. Similar to Figure 3 but for observations of NML Cyg. For each of the observed wavebands, we do not resolve any obvious excess emission above the PSF.

As noted in Schuster et al. (2009), the MIRAC images do indeed appear asymmetric along the NW-SE axis. For these images, we generate isophotes and separate our radial profiles into two axes. The major axis (NW-SE) is shown with solid points in Figure 14, and the minor axis (NE-SW) is shown with open circles. Here, we see that the constant mass-loss, $q=2$, 

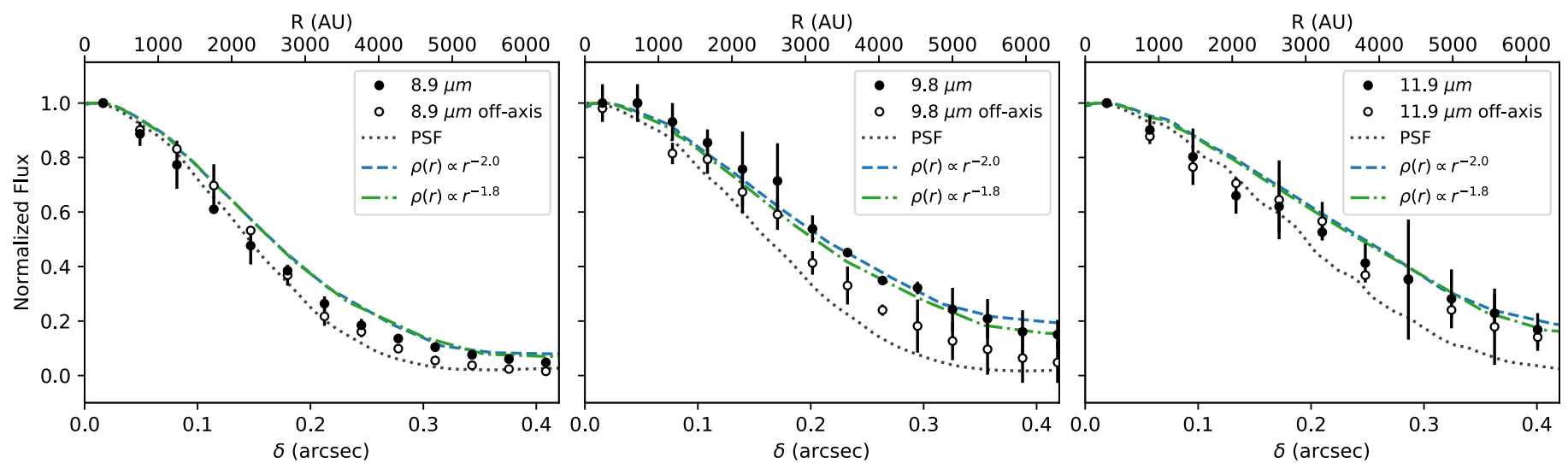

Figure 14. Radial profiles of NML Cyg from the three MIRAC filters, reproduced from Schuster et al. (2009). Due to the observed asymmetry from interactions with $\mathrm{Cyg} \mathrm{OB} 2$, we have divided the surface brightness measurements into two different axes. Isophotal analysis confirms the elongation along the major (NE-SW) axis, shown with solid points. The minor axis is shown with open circles.

model does fit the observed major-axis brightness profile for the $9.8 \mu \mathrm{m}$ image, though the $q=1.8$ profile is similar in shape for all three MIRAC bands. We note that DUSTY cannot take into account the external radiation field from Cyg OB2, so the model profile shapes are not necessarily conclusive as to the mass-loss history of NML Cyg.

NML Cyg has one of the highest mass-loss rates of any RSG/hypergiant-from $6.4 \times 10^{-5} M_{\odot} \mathrm{yr}^{-1}$ (Morris \& Jura 1983) to as high as $1.6 \times 10^{-4} M_{\odot} \mathrm{yr}^{-1}$ (Lucas et al. 1992). We calculate even higher mass-loss rates from the DUSTY models, though, at $4-5 \times 10^{-4} M_{\odot} \mathrm{yr}^{-1}$ with average outflow velocity $23 \mathrm{~km} \mathrm{~s}^{-1}$ (Schuster et al. 2009). It is likely, however, that the complex morphology of NML Cyg cannot be well-modeled with a single-component power-law dust mass distribution, similar to the difficulties in modeling $\mathrm{S}$ Per with its known asymmetric profile.

\section{Conclusions}

With mid-infrared imaging from MMT/MIRAC and SOFIA/FORCAST, we observed three OH/IR RSGs, NML Cyg, VX Sgr, and S Per and the normal RSGs RS Per and $\mathrm{T}$ Per. We present new photometry at $9-11 \mu \mathrm{m}$ with MIRAC, at 20-40 $\mu \mathrm{m}$ with FORCAST, and at 70 and $160 \mu \mathrm{m}$ from the Herschel/PACS archive. These data, in combination with published optical and near- to mid-IR photometry, are used to constrain DUSTY model SEDs.

VX Sgr: Though a symmetric extended circumstellar envelope is resolved in HST images (Schuster et al. 2006), we have only marginally resolved a cooler dust component at 19.7-37.1 $\mu \mathrm{m}$ with FORCAST. From DUSTY, we conclude that the mass loss around VX Sgr cannot necessarily be wellmodeled by smooth, constant mass loss. The IR excess emission combined with DUSTY modeling show evidence for a higher mass-loss rate in the past for VX Sgr with an average estimated mass-loss rate of $2 \times 10^{-5} M_{\odot} \mathrm{yr}^{-1}$ for the $\rho(r) \propto r^{-1.6}$ model, and $5 \times 10^{-5} M_{\odot} \mathrm{yr}^{-1}$ for the constant mass-loss case.

$S$ Per: Azimuthal profiles of S Per reveal an IR excess above the PSF emission from the central star in MIRAC imaging and to a lesser extent in the FORCAST wavelengths. However, the radial profiles produced from DUSTY are not significantly different when convolved with the optics of SOFIA. Both the $r^{-1.6}$ dust density distribution model and the enhanced massloss model fit the near- to mid-IR SED out to $160 \mu \mathrm{m}$, implying the possibility of a higher mass-loss rate in the past. S Per is known to posses an asymmetric outflow close to the central star and lack an extended spherical nebula; therefore, the 1D spherically symmetric radiative-transfer code DUSTY may not be the most accurate method for reconstructing the mass-loss history of S Per. We estimate an average mass-loss rate of $\sim 2-3 \times 10^{-5} M_{\odot} \mathrm{yr}^{-1}$.

RS Per and T Per: SED models of both stars suggest that a constant mass-loss rate is insufficient to generate enough 20-40 $\mu \mathrm{m}$ emission to match the observed mid-IR photometry. The FORCAST images for both RSGs, and the MIRAC $10 \mu \mathrm{m}$ image for T Per, show modest excess emission above the flux from the PSF. The SED for RS Per appears to be best fit with a shallow power-law distribution in dust density of $\rho(r) \propto r^{-1.6}$, suggesting it had a higher mass-loss rate in the past. Over the lifetimes of the observed dust shells, we estimate average massloss rates of $4 \times 10^{-5} M_{\odot} \mathrm{yr}^{-1}$ for RS Per and $8 \times$ $10^{-6} M_{\odot} \mathrm{yr}^{-1}$ for $\mathrm{T}$ Per.

NML Cyg: We do not observe any circumstellar envelope around NML Cyg at 31.5 and $37.1 \mu \mathrm{m}$. Though the DUSTY constant mass-loss models appear to fit the near- to far-IR SED accurately, we cannot conclude from these data alone whether the mass loss around NML Cyg is smooth and constant, or decreasing over time. Additionally, at the resolution of FORCAST at 20-40 $\mu \mathrm{m}$, we do not observe any evidence for an optically thick, cool dust shell. The DUSTY models provide an estimate for a mass-loss rate of $\sim 4-5 \times 10^{-4} M_{\odot} \mathrm{yr}^{-1}$. Finally, as described in Schuster et al. (2006, 2009), there is an external heat source affecting the temperature structure of the circumstellar envelope surrounding NML Cyg. As DUSTY can only model sources with central internal heating, discrepancies in the mass-loss rates from different measurements probing various parts of the envelope are expected.

In Figure 15, we summarize the results from this work and Paper I. We plot the estimated mass-loss rates as a function of luminosity with three mass-loss rate prescriptions from Mauron \& Josselin (2011). Reimers (1975) and Kudritzki \& Reimers (1978) measured mass-loss rates for O-rich dust-enshrouded RSGs in the LMC and fit an empirical relation to luminosity. Reimers' law is largely consistent with the later formulations, NJ90 (Nieuwenhuijzen \& de Jager 1990) and observations by van Loon et al. (2005) on dusty RSGs in the LMC at lower luminosities $\left(L \lesssim 2 \times 10^{5} L_{\odot}\right)$. Mauron \& Josselin (2011) apply these mass-loss rate prescriptions to a number of Galactic 


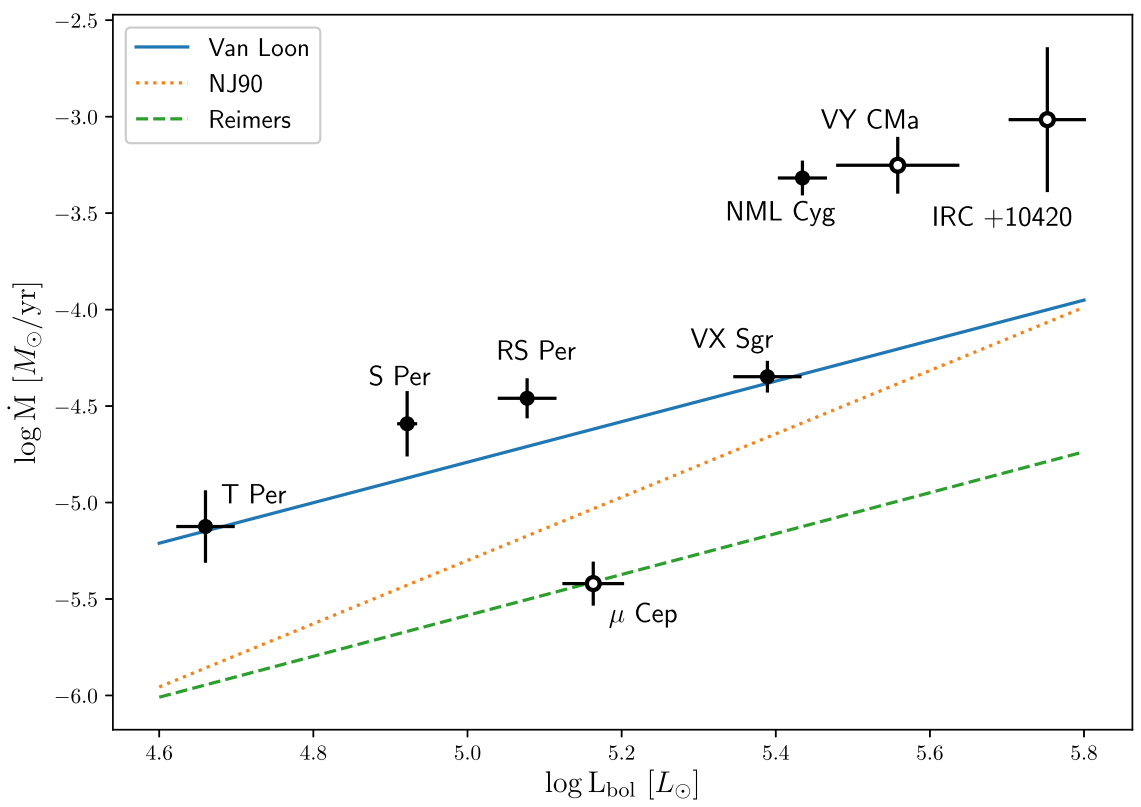

Figure 15. Average mass-loss rates as a function of luminosity. Stars with open circles are the sources discussed in Paper I. Luminosity values (and luminosity error bars) are drawn from the literature (see Section 4). Overplotted are mass-loss prescriptions for dusty RSGs from van Loon et al. (2005), Nieuwenhuijzen \& de Jager (1990) (NJ90), and Reimers Law (Reimers 1975; Kudritzki \& Reimers 1978). Each of the models displayed are calculated with fixed stellar effective temperature of $3750 \mathrm{~K}$ for consistency.

RSGs. In Figure 15, we reproduce their implemented formulae at a fixed stellar effective temperature of $3750 \mathrm{~K}$ for consistency with their figures. We adopt their luminosities for all of the sources except IRC $+10420\left(\log L / L_{\odot} \approx 5.7-5.8\right.$, De Beck et al. 2010; Tiffany et al. 2010). The error bars shown in the $y$-axis (mas-loss rate) are the standard deviation of the derived mass-loss rates from the five best-fitting DUSTY models, and the error bars in the $x$-axis (luminosity) are from the literature.

We note that the mass-loss rates for the stars in our sample are largely consistent with the Van Loon and NJ90 prescriptions (analytical forms given in Mauron \& Josselin 2011). As noted in Paper I, $\mu$ Cep has a curiously low mass-loss rate for its luminosity class, and the hypergiants VY CMa and IRC +10420 are well-known, extremely luminous hypergiants whose mass-loss rates are among the highest observed.

DUSTY modeling, in combination with imaging from SOFIA/FORCAST and MIRAC, are powerful tools for exploring the mass-loss histories and dust density profiles of luminous supergiants. Although the spatial resolution of FORCAST yielded a PSF too wide for us to compare DUSTY output radial profiles, the photometry from 20 to $40 \mu \mathrm{m}$ represents a crucial data set in constraining the thermal dust properties of RSGs. The models shown here and in Paper I hint at possible variable mass-loss rates among the most luminous RSGs while others have constant mass-loss histories over the past few thousand years. We plan to follow up observations of the supergiants discussed here with high-resolution imaging at 5-12 $\mu \mathrm{m}$ with LMIRCam and NOMIC on the LBT. Surface brightness profiles with better spatial resolution at these shorter wavelengths, when coupled with SED modeling, will allow us to characterize mass-loss events from the last few hundred years.

We thank Rubab Khan for discussion on $\chi^{2}$-minimization of DUSTY models. This work has used unpublished data from Michael Schuster's PhD thesis, which is available through the
SAO/NASA Astrophysics Data System (ADS) at http:// adsabs.harvard.edu/abs/2007PhDT........28S. Financial support for this work was provided by NASA through awards SOF 030082 and SOF 040013 to R M Humphreys issued by USRA. R.D.G. acknowledges support from NASA and the United States Air Force.

Facilities: Akari, Herschel (PACS), IRAS, ISO, MMT (MIRAC), Spitzer, SOFIA (FORCAST).

Software: Astropy (Astropy Collaboration et al. 2013), DUSTY (Ivezic et al. 1997), HIPE (Ott et al. 2010).

\section{ORCID iDs}

Michael S. Gordon (1D https://orcid.org/0000-0002-1913-2682 Roberta M. Humphreys (ib https://orcid.org/0000-00031720-9807

Terry J. Jones (iD https://orcid.org/0000-0002-8716-6980

Dinesh Shenoy (iD https://orcid.org/0000-0002-2398-145X

Robert D. Gehrz (iD https://orcid.org/0000-0003-1319-4089

Massimo Marengo (iD https://orcid.org/0000-0001-9910-9230

\section{References}

Abrahamyan, H. V., Mickaelian, A. M., \& Knyazyan, A. V. 2015, A\&C, 10,99

Anderson, E., \& Francis, C. 2012, AstL, 38, 331

Aoki, W., Tsuji, T., \& Ohnaka, K. 1998, A\&A, 340, 222

Asaki, Y., Deguchi, S., Imai, H., et al. 2010, ApJ, 721, 267

Astropy Collaboration, Robitaille, T. P., Tollerud, E. J., et al. 2013, A\&A, 558, A33

Baron, F., Monnier, J. D., Kiss, L. L., et al. 2014, ApJ, 785, 46

Biller, B. A., Close, L. M., Li, A., et al. 2005, ApJ, 620, 450

De Beck, E., Decin, L., de Koter, A., et al. 2010, A\&A, 523, A18

de Graauw, T., Haser, L. N., Beintema, D. A., et al. 1996, A\&A, 315, L49

de Jager, C., Nieuwenhuijzen, H., \& van der Hucht, K. A. 1988, A\&AS, 72,259

Decin, L., Hony, S., de Koter, A., et al. 2006, A\&A, 456, 549

Egan, M. P., Price, S. D., \& Kraemer, K. E. 2003, BAAS, 35, 1301

Fok, T. K. T., Nakashima, J.-i., Yung, B. H. K., Hsia, C.-H., \& Deguchi, S 2012, ApJ, 760, 65

Gehrz, R. D., \& Woolf, N. J. 1971, ApJ, 165, 285 
Groenewegen, M. A. T. 2012, A\&A, 540, A32

Groenewegen, M. A. T., Waelkens, C., Barlow, M. J., et al. 2011, A\&A, 526, A162

Henden, A. 2016, JAVSO, 44, 84

Heras, A. M., \& Hony, S. 2005, A\&A, 439, 171

Herter, T. L., Adams, J. D., De Buizer, J. M., et al. 2012, ApJL, 749, L18

Herter, T. L., Vacca, W. D., Adams, J. D., et al. 2013, PASP, 125, 1393

Hinz, P. M., Angel, J. R. P., Woolf, N. J., Hoffmann, W. F., \& McCarthy, D. W. 2000, Proc. SPIE, 4006, 349

Hoffmann, W. F., Hinz, P. M., Defrère, D., et al. 2014, Proc. SPIE, 9147, 914710

Hoffmann, W. F., Hora, J. L., Fazio, G. G., Deutsch, L. K., \& Dayal, A. 1998, Proc. SPIE, 3354, 647

Humphreys, R. M. 1978, ApJS, 38, 309

Humphreys, R. M., Davidson, K., Ruch, G., \& Wallerstein, G. 2005, AJ, 129,492

Humphreys, R. M., Helton, L. A., \& Jones, T. J. 2007, AJ, 133, 2716

Humphreys, R. M., \& Lockwood, G. W. 1972, ApJL, 172, L59

Humphreys, R. M., Smith, N., Davidson, K., et al. 1997, AJ, 114, 2778

Ivezic, Z., Groenewegen, M. A. T., Men'shchikov, A., \& Szczerba, R. 1997, MNRAS, 291, 121

Jiang, B. W., Deguchi, S., \& Ramesh, B. 1999, PASJ, 51, 95

Jura, M., \& Kleinmann, S. G. 1990, ApJS, 73, 769

Justtanont, K., de Jong, T., Helmich, F. P., et al. 1996, A\&A, 315, L217

Kiss, L. L., Szabó, G. M., \& Bedding, T. R. 2006, MNRAS, 372, 1721

Knapp, G. R., \& Morris, M. 1985, ApJ, 292, 640

Knapp, G. R., Sandell, G., \& Robson, E. I. 1993, ApJS, 88, 173

Kudritzki, R. P., \& Reimers, D. 1978, A\&A, 70, 227

Lucas, R., Bujarrabal, V., Guilloteau, S., et al. 1992, A\&A, 262, 491

Mathis, J. S., Rumpl, W., \& Nordsieck, K. H. 1977, ApJ, 217, 425

Matt, S., Balick, B., Winglee, R., \& Goodson, A. 2000, ApJ, 545, 965

Mauron, N., \& Josselin, E. 2011, A\&A, 526, A156

Morris, M., \& Jura, M. 1983, ApJ, 267, 179

Myers, J. R., Sande, C. B., Miller, A. C., Warren, W. H., Jr., \& Tracewell, D. A. 2015, yCat, 5145, 0

Nieuwenhuijzen, H., \& de Jager, C. 1990, A\&A, 231, 134
O’Donnell, J. E. 1994, ApJ, 422, 158

Ossenkopf, V., Henning, T., \& Mathis, J. S. 1992, A\&A, 261, 567

Ott, S. 2010, in ASP Conf. Ser. 434, Astronomical Data Analysis Software and Systems XIX, ed. Y. Mizumoto, K.-I. Morita, \& M. Ohishi (San Francisco, CA: ASP), 139

Pilbratt, G. L., Riedinger, J. R., Passvogel, T., et al. 2010, A\&A, 518, L1

Poglitsch, A., Waelkens, C., Geis, N., et al. 2010, A\&A, 518, L2

Price, S. D., Smith, B. J., Kuchar, T. A., Mizuno, D. R., \& Kraemer, K. E. 2010, ApJS, 190, 203

Reimers, D. 1975, MSRSL, 8, 369

Richards, A. M. S., Yates, J. A., \& Cohen, R. J. 1999, MNRAS, 306, 954

Schuster, M. T. 2007, PhD thesis, Univ. Minnesota

Schuster, M. T., Humphreys, R. M., \& Marengo, M. 2006, AJ, 131, 603

Schuster, M. T., Marengo, M., Hora, J. L., et al. 2009, ApJ, 699, 1423

Shenoy, D., Humphreys, R. M., Jones, T. J., et al. 2016, AJ, 151, 51

Shenoy, D. P., Jones, T. J., Humphreys, R. M., et al. 2013, AJ, 146, 90

Shenoy, D. P., Jones, T. J., Packham, C., \& Lopez-Rodriguez, E. 2015, AJ, 150,15

Skemer, A. J., Close, L. M., Hinz, P. M., et al. 2008, ApJ, 676, 1082

Skrutskie, M. F., Jones, T., Hinz, P., et al. 2010, Proc. SPIE, 7735, 77353H

Smartt, S. J. 2015, PASA, 32, e016

Smartt, S. J., Eldridge, J. J., Crockett, R. M., \& Maund, J. R. 2009, MNRAS, 395, 1409

Smith, B. J., Price, S. D., \& Baker, R. I. 2004, ApJS, 154, 673

Smith, N., Humphreys, R. M., Davidson, K., et al. 2001, AJ, 121, 1111

Speck, A. K., Barlow, M. J., Sylvester, R. J., \& Hofmeister, A. M. 2000 , A\&AS, 146, 437

Tiffany, C., Humphreys, R. M., Jones, T. J., \& Davidson, K. 2010, AJ, 140,339

van Loon, J. T., Cioni, M.-R. L., Zijlstra, A. A., \& Loup, C. 2005, A\&A, 438, 273

Vlemmings, W., Diamond, P. J., \& van Langevelde, H. J. 2001, A\&A, 375, L1

Vlemmings, W. H. T., van Langevelde, H. J., \& Diamond, P. J. 2005, A\&A, 434, 1029

Wright, E. L., Eisenhardt, P. R. M., Mainzer, A. K., et al. 2010, AJ, 140, 1868 